\title{
Tabularia
}

TABULARIA Sources écrites des mondes normands médiévaux Guillaume de Volpiano : Fécamp et l'histoire normande | 2004

\section{Une reconstitution hypothétique du cheminement des Annales de Flodoard, depuis Reims jusqu'à Fécamp}

From Reims to Fécamp, an hypothetical reconstruction of the route of the Annals of Flodoard

\section{Stéphane Lecouteux}

\section{OpenEdition}

\section{Journals}

Édition électronique

URL : http://journals.openedition.org/tabularia/1923

DOI : $10.4000 /$ tabularia. 1923

ISSN : 1630-7364

Éditeur :

CRAHAM - Centre Michel de Boüard, Presses universitaires de Caen

\section{Référence électronique}

Stéphane Lecouteux, « Une reconstitution hypothétique du cheminement des Annales de Flodoard depuis Reims jusqu'à Fécamp ", Tabularia [En ligne], Guillaume de Volpiano : Fécamp et l'histoire normande, mis en ligne le 15 janvier 2004, consulté le 19 avril 2019. URL : http:// journals.openedition.org/tabularia/1923; DOI : 10.4000/tabularia.1923 


\title{
Une reconstitution hypothétique du cheminement des Annales de Flodoard, depuis Reims jusqu'à Fécamp \\ From Reims to Fécamp, an hypothetical reconstruction of the route of the Annals of Flodoard
}

\author{
Stéphane LECOUTEUX \\ 37, av. du Grand Châtelet \\ 38100 Grenoble \\ lecouteux.stephmanue@wanadoo.fr
}

Résumé :

Il est aujourd'hui admis que Dudon de Saint-Quentin eut recours aux Annales de Flodoard pour rédiger son histoire des premiers ducs de Normandie. Leur introduction à Fécamp est généralement attribuée au réformateur clunisien, Guillaume de Volpiano, peu après l'An mil. Cependant, cette dernière hypothèse est en réalité peu probable pour diverses raisons, notamment chronologiques. Le cheminement des Annales depuis Reims, où Flodoard rédigea son manuscrit original, jusqu'à Fécamp, où elles firent souche pour constituer «la branche normande» de cette œuvre, demeure donc relativement obscur. Afin de tenter sa reconstitution, un réexamen de plusieurs manuscrits décrits par Philippe Lauer dans son édition des Annales s'impose. Au cours de cette étude, une identification du copiste ayant introduit le premier manuscrit en Normandie sera envisagée et une hypothèse sera émise au sujet «du continuateur » de ces Annales sur la période 966-978, jusqu'ici resté anonyme. Certaines constatations ouvrent de nouvelles perspectives pour la localisation des lieux d'éducation et de formation de Dudon.

Mots-clés: Annales de Flodoard, Fécamp, Dudon de Saint-Quentin, Guillaume de Volpiano, Philippe Lauer, Richard de Normandie, Adalbéron, Roricon, Laon, Reims.

Abstract:

It is well known that Dudo of Saint-Quentin used the Annals of Flodoard to write his story of the first dukes of Normandy. The Annals' presence in Fécamp is generally attributed to the reformer of Cluny, William of Volpiano, just after year 1000. However, for reasons of chronology this assumption cannot be sustained. Flodoard wrote the original manuscript of the annals at Reims, and the route by which his Annals came to Fécamp, to become "the Norman branch » of this work, remains relatively obscure. In order to reconstruct this route, a closer study of the manuscripts, first analyzed by the Annals' editor Philippe Lauer, will be presented here. This analysis will suggest an identification of the copyist who may have introduced the first manuscript of Flodoard's Annals in Normandy. It will also make new suggestions as to the identity of the anonymous continuator for the period 966-978. New leads are suggested, too, with regard to Dudo's education and the schools where he may have been taught.

Keywords: Annals of Flodoard, Fécamp, Dudo of Saint-Quentin, William of Volpiano, Philippe Lauer, Richard I of Normandy, Richard II of Normandy, Adalbero, Rorico, Laon, Reims. 
Dans son édition de Dudon de Saint-Quentin, Jules Lair ${ }^{1}$ affirmait - sans véritable preuve - que le chanoine de Saint-Quentin n'avait pu utiliser comme source les Annales de Flodoard. Paradoxalement, il mettait en évidence de nombreux faits pouvant être rapprochés entre ces deux œuvres. Par la suite, Philippe Lauer ${ }^{2}$ indiqua au contraire la forte probabilité d'une connaissance par Dudon d'une copie des Annales présente en Normandie dès le début du XI ${ }^{\mathrm{e}}$ siècle. Enfin, Henri Prentout, dans son étude critique ${ }^{3}$, démontra l'utilisation par le chanoine des Annales de Flodoard sur l'ensemble de la période couverte par celles-ci (919-966). Ce fait est aujourd'hui reconnu de façon unanime et la version des Annales utilisée par Dudon est généralement identifiée comme celle introduite à Fécamp par Guillaume de Volpiano.

\section{Les Annales de Flodoard ont-elles été amenées en Normandie par Guillaume de Volpiano?}

Philippe Lauer a émis l'hypothèse selon laquelle «la branche normande» des Annales de Flodoard aurait pour source un manuscrit apporté par le réformateur clunisien, Guillaume de Volpiano, connu également sous le nom de Guillaume de Dijon ${ }^{4}$. Ce dernier, sollicité par le duc Richard II pour réformer l'abbaye de Fécamp vers 1001, aurait introduit les Annales en Normandie à cette occasion. Du fait de découvertes plus ou moins récentes, cette hypothèse n'est pas sans cacher plusieurs zones d'ombre, ni soulever quelques interrogations.

Connu pour l'efficacité de ses techniques de réforme et de rétablissement de l'ordre selon les méthodes clunisiennes, c'est dans cette unique optique que Guillaume de Volpiano fut vraisemblablement appelé à Fécamp par le duc de Normandie ${ }^{5}$. Nous savons en particulier que ce grand réformateur instruisait ses élèves en insistant sur trois aspects : le respect de la règle de Saint-Benoît (prioritaire), l'apprentissage de la lecture et, enfin, la formation au chant (objectif ultime une fois les deux autres acquis) ${ }^{6}$. Les Annales de Flodoard ne semblent pas, a priori, l'outil de travail idéal pour atteindre l'un de ces objectifs. Le genre annalistique est même en total

1. Jules Lair (DUDON DE SAINT-QUENTIN, p. 115-314) prétend que Dudon ne pouvait connaître les annales majeures carolingiennes, dont celles de Flodoard. Dudon connaissait pourtant visiblement cette œuvre (voir l'allusion à la bataille de Soissons, p. 173).

2. LAUER, 1898, p. 521-523; FLODOARD, p. XXXVIII-XLII. On notera que dans l'introduction de son édition des Annales de Flodoard, Philippe Lauer se réfere à son étude détaillée du manuscrit 633 du Vatican, et apporte quelques corrections à celle-ci.

3. Prentout, 1916, p. 26-27. Si cette étude de l'œuvre de Dudon est critiquable sur de nombreux points - à cause notamment du jugement draconien porté sur le chanoine de Saint-Quentin - reconnaissons cependant à Henri Prentout le mérite d'avoir identifié une partie des sources utilisées par Dudon pour constituer son œuvre. L'utilisation des Annales de Flodoard par Dudon fait partie des démonstrations importantes faites par cet auteur.

4. FlodoARD, p. XLVII-XLIX et XLII.

5. GAZEAU, 2002, p. 37-38.

6. «Le B. Guillaume, abbé de S. Bénigne de Dijon », in Histoire Littéraire de la France, t. VII, p. 318-325. 
décalage avec l'enseignement visé, ainsi qu'avec les manuels d'étude et d'apprentissage communément utilisés à cet effet. De plus, pourquoi les avoir amenées précisément à Fécamp, et non dans les nombreux autres monastères réformés par ses soins? Ce serait en effet, à notre connaissance, la seule abbaye où Guillaume de Volpiano aurait apporté une copie des Annales. Flodoard relate pourtant des événements sur la plupart des régions où Guillaume fit ses réformes ${ }^{7}$, et on devine assez mal pourquoi seule la Normandie - et Fécamp - aurait eu le privilège de recevoir une copie de cette œuvre: en effet, l'abbé de Dijon n'avait apparemment pas plus de raisons d'apporter ce manuscrit à l'abbaye de Fécamp que dans l'un des nombreux autres monastères réformés par ses soins...

L'analyse chronologique des faits suscite une autre interrogation. On pense aujourd'hui que l'œuvre de Dudon était déjà bien avancée lorsque survint la mort de Richard I $^{\text {er }}$ de Normandie en 996. En effet, une première version était certainement terminée peu de temps après l'accès au pouvoir de Richard II, c'est-à-dire à la fin du premier millénaire ${ }^{8}$. À cette époque, le chanoine de Saint-Quentin avait donc déjà eu recours aux Annales depuis au moins quelques années. Si, selon l'hypothèse de Philippe Lauer, celle-ci n'était parvenue en Normandie qu'avec Guillaume de Volpiano, c'est-à-dire en 1001 au plus tôt, comment Dudon aurait-il pu utiliser la première copie normande des Annales de Flodoard comme source pour son œuvre dans ces conditions?

Un dernier fait pour le moins singulier ne joue pas en faveur de cette hypothèse. Guillaume de Volpiano sollicita le moine Raoul Glaber pour qu'il rédige une histoire des événements survenus en Occident autour de l'an mil ${ }^{9}$. Or ce dernier n'eût manifestement aucun recours aux Annales de Flodoard dans son ouvrage. Son objectif était de faire débuter son récit vers $900^{10}$ et les Annales - couvrant les années 919 à

7. Gazeau, Véronique, "Guillaume de Volpiano", in Dictionnaire du Moyen-Âge, p. 643. RaOul GLABER, trad. ARNOUX, p. 9. « Le B. Guillaume, abbé de S. Bénigne de Dijon », in Histoire Littéraire de la France, t. VII, p. 318-325. Depuis la réforme de l'abbaye Saint-Bénigne de Dijon jusqu'à sa mort en 1031, Guillaume de Volpiano réformera, plus ou moins directement, de trente à quarante monastères en Bourgogne, Lotharingie, Normandie et Italie. Or, Flodoard relate dans ses Annales de nombreux faits sur ces régions et pays.

8. BOUET, 2002 (p. 58-59 et 66) et ARNOUX, 1999, p. 41, estiment que la plus grande partie du travail de Dudon a déjà été exécutée au commencement du règne de Richard II. Le ms de Berne, qui ne contient que le texte en prose sans la préface dédicatoire et les poèmes en vers, est probablement le manuscrit le plus proche de cette première version. Le ms disparu de Saint-Évroult était lui aussi dépourvu de ces pièces de vers (PRENTOUT, 1916, p. 25). L'ajout des poèmes semble avoir eu lieu progressivement par la suite, si bien que certains manuscrits en comptent davantage que d'autres: $c$ 'est le cas notamment du ms de Berlin, qui comporte un poème final sur Fécamp et Jean de Ravenne. Dudon semble donc avoir apporté régulièrement des additions à sa version initiale, même après 1015 , à l'époque où il devint doyen de Saint-Quentin et rédigea sa préface dédicatoire (SHOPKOW, 1989 , p. 36, n 27$)$

9. Raoul Glaber, trad. Mathieu Arnoux, p. 10, 36 n $^{\circ} 1$ et 175 ; RaOUl Glaber, éd. Prou, p. V-VII.

10. Même si Raoul Glaber se montre plus prolixe sur les événements contemporains concernant les années 1010-1040 (trad. ARNOUX, p. 17), il indique rapporter des faits survenus entre 900 et son époque (ibid. I, 4, p. 45). La rédaction de ses cinq livres eut lieu entre 1016 - livre II - et 1047 livre V (ibid., p. 13). 
966 - auraient été une source précieuse pour lui: en plus des événements historiques, les miracles et phénomènes météorologiques relatés par Flodoard auraient certainement eu une place de choix dans son récit. Il en ressort que Raoul Glaber n'a visiblement jamais eu connaissance de cette œuvre ${ }^{11}$. Si Guillaume de Volpiano l'avait luimême connue, il n'aurait pas manqué de la mettre entre les mains de son élève afin qu'il accomplisse au mieux la tâche demandée. Ce qui laisse supposer qu'entre 1026 et 1028, alors que Raoul Glaber accompagnait l'abbé de Dijon en Italie, aucun d'eux ne connaissait encore l'existence des Annales ${ }^{12}$. À cette époque, l'œuvre de Dudon était déjà achevée depuis longtemps, et il restait à Guillaume de Volpiano moins de trois années à vivre.

Tout ceci laisse suspecter une introduction du premier manuscrit des Annales de Flodoard en Normandie par une autre personne que Guillaume de Volpiano. À moins que Dudon ait lui-même utilisé un autre manuscrit ailleurs que dans cette région pour constituer son œuvre. La question de l'identification du manuscrit des Annales employé par Dudon se pose alors.

\section{Analyse et étude des manuscrits de « la branche normande»}

Cette constatation nous conduit à analyser en détail les différents manuscrits décrits par Philippe Lauer ${ }^{13}$. Ce dernier les répartit en deux principaux groupes: le premier ne contient que le manuscrit de Dijon $\left(\mathrm{ms} \mathrm{A}^{14}\right)$, marginal par rapport à tous

11. Plusieurs détails montrent que Raoul Glaber ne connaissait probablement pas les Annales et eut recours à d'autres sources pour rédiger ses Histoires: il mentionne l'exil de Louis IV outre-Rhin et non outre-mer (trad. ARNOUX, I, 5, p. 51; FLODOARD, a. 936, p. 63), l'annexion de la Lotharingie par Otton I ${ }^{\text {er }}$, alors que son père Henri avait rallié les Lorrains au royaume de Germanie dès 925 (trad. ARNOUX, I, 7, p. 53; FlODOARD, a. 925, p. 32) et il indique que le roi Robert fut tué à Soissons par les Saxons et leur roi Otton I $^{\text {er }}$ plutôt que par l'armée de Charles le Simple jointe aux Lorrains (ibid., I, 6, p. 51 et III, 39, p. 215-217; FlODOARD, a. 923, p. 13). Par ailleurs, il rapporte de façon différente la capture de Charles le Simple par Herbert de Vermandois (ibid., I, 5, p. 49; FlodOARD, a. 923, p. 15), la mort de ce dernier (ibid., I, 7, p. 53; FlODOARD, a. 943, p. 86), le choix de Raoul comme roi par Hugues le Grand (ibid., I, 6, p. 51; FlODOARD, a. 923, p. 14), ou encore l'assassinat de Guillaume Longue-Épée, imputé surtout à Thibaut le Tricheur (ibid., III, 39, p. 215-216; FLODOARD, a. 943 , p. 86).

12. RaOUl Glaber, trad. Arnoux, p. 9 et 11 ; RaOul Glaber, éd. Prou, p. VI. Le fait que Guillaume de Volpiano n'ait pas eu connaissance de cette œuvre malgré les très nombreuses abbayes qu'il réforma à travers la France entre 989 et 1031 peut paraître étrange. Il en va de même pour Raoul Glaber, qui parcourut de nombreux monastères de Bourgogne dépendants de Cluny entre 1000 et 1047 (éd. Mathieu ARNOUX, p. 8 et 10-11). Il semble en effet que cette œuvre de Flodoard soit arrivée tardivement dans les monastères dépendants de cet ordre : le fait que l'œuvre soit issue de Reims, dont l'école forma autour de l'an mil quelques-uns des plus vifs opposants aux clunisiens (notamment Adalbéron de Laon et probablement Gérard de Cambrai) en fut peut-être l'une des causes...

13. Pour identifier ces manuscrits, je reprends ici les lettres latines majuscules utilisées pour leur description par Philippe Lauer dans son édition des Annales (FLODOARD, p. XXXII-XLV). J'utilise en revanche des lettres grecques pour identifier les manuscrits aujourd'hui disparus.

14. FlodoARD, p. XXXII-XXXV (ms A). 
les autres ${ }^{15}$. Le second groupe ( $\mathrm{ms} \mathrm{B}^{16}, \mathrm{C}, \mathrm{D}, \mathrm{E}, \mathrm{F}$ et $\left.\mathrm{G}\right)$ contient des manuscrits issus d'un ancêtre commun disparu, dû à un continuateur des Annales (appelons-le $\alpha$ ): les manuscrits de ce groupe comportent à la fin une rubrique Hucusque Cronica Flodoardi Presbiteri précédant des additions postérieures à la mort de Flodoard survenue en 966. En poussant son analyse plus loin, Philippe Lauer définit dans son second groupe, un sous-groupe constitué uniquement de $\mathrm{C}, \mathrm{D}$, et $\mathrm{E}^{17}$ : ces trois manuscrits dérivent tous d'un ancêtre commun lui aussi disparu (appelons-le $\beta$ ), et forment «la branche normande» des Annales de Flodoard. Ce sont eux que nous allons étudier ici :

C. - Manuscrit $633^{2}$ Reg. (fonds de la reine Christine) de la Bibliothèque Vaticane $^{18}:$ ms de Fécamp.

Écrit au début du XI ${ }^{\mathrm{e}}$ siècle, son folio 80r renferme des notes du XII ${ }^{\mathrm{e}}$ siècle sur Saint-Taurin d'Evreux, indiquant sa présence en Normandie à cette époque. Il s'y trouvait encore au $\mathrm{XV}^{\mathrm{e}}$ siècle: une note montre qu'alors il était à Fécamp. Il porte en titre la rubrique Incipit Gesta Francorum, mais Philippe Lauer a constaté que le texte avait été gratté. Par une brillante démonstration, il a prouvé que la rubrique initiale était Incipit Gesta Normannorum et que le grattage avait probablement eu lieu vers le XII ${ }^{\mathrm{e}}$ siècle, lorsque les Annales de Fulda furent mises en tête de celles de Flodoard ${ }^{19}$. Ce titre initial renforce l'idée d'une origine normande de ce manuscrit. Au-dessus, une main plus récente a ajouté les mots Chronica Flodoardi Presbyteri ${ }^{20}$.

15. Pour l'examen des différents manuscrits, cf. FLODOARD, p. XLV-XLVII.

16. FlODOARD, p. XXXV-XXXVIII (ms B).

17. Philippe Lauer exclut de son étude les manuscrits $F$ et $G$, jugés peu intéressants car copiés respectivement sur $\mathrm{B}$, et sur $\mathrm{C}, \mathrm{D}$ ou $\mathrm{E}$ à partir du $\mathrm{XV}^{\mathrm{e}}$ siècle. N'ayant pas toutes les informations à leur sujet, nous devons nous résigner à les écarter nous aussi de notre étude, bien que $\mathrm{G}$ dérive lui aussi de la «branche normande». Voyez FlodoARD, p. XLIV - XLV (ms F et G).

18. FlodoARd, p. XXXVIII-XLII (ms C). LAUER, Philippe, «Le manuscrit des Annales de Flodoard, reg. lat. 633 du Vatican», p. 491 et suiv. Ce ms porte aujourd'hui la référence Vat. Reg. lat. 994 (SOT, Michel, p. 74).

19. FlODOARD, p. L et LXX (fac-similé C). LAUER, 1898, p. 503-504 et planche XIII (fac-similé). Pour expliquer les notes du folio 80r, Philippe Lauer pense que ce manuscrit a été apporté au monastère de Saint-Taurin d'Evreux par Guillaume de Volpiano, qui s'y serait retiré en 1028, puis y aurait été enterré en 1031. Tout d'abord, c'est à Fécamp que Guillaume est décédé (GAZEAU, 2002, p. 37) et fut enterré en 1031 (BROCKHAUs, 2002, p. 73-74). Ensuite, c'est seulement sous Robert le Magnifique et l'abbé Jean de Ravenne, en 1034, que le monastère de Saint-Taurin fut rattaché à l'abbaye de Fécamp (GAZEAU, 2002, p. 40 ; BROCKHAUS, 2002, p. 76). Enfin, un office dédié à Saint-Taurin fut composé à Fécamp, après la mort de Guillaume (GAZEAU, 2002, p. 41) : les notes du XII ${ }^{\mathrm{e}}$ siècle du folio 80r semblent tirées de cet office. On notera également qu'une Vita sancti Taurini figure dans le plus ancien catalogue de l'abbaye de Fécamp. Le manuscrit $\mathrm{C}$ a donc probablement été copié à Fécamp et n'a jamais quitté cette ville avant le $\mathrm{XV}^{\mathrm{e}}$ siècle, comme le suggère les notes du folio $80 \mathrm{v}$ d'une main de cette époque.

20. FlODOARD, p. LXX (fac-similé C). LAUER, 1898, planche XIII (fac-similé). 
D. - Manuscrit lat. 5354 de la Bibliothèque nationale ${ }^{21}$ : ms de Bonneval.

Ce manuscrit date du XI ${ }^{\text {e }}$ siècle, probablement de la première moitié. Les notes $\mathrm{du}$ folio $139 \mathrm{v}$ prouvent sa présence au monastère de Bonneval - diocèse de Chartres - au XIII e siècle. Il porte le titre Incipit cronica Frodoardi, presbiteri, de gestis Normannorum, ce qui laisse là aussi envisager une origine normande.

E. - Manuscrit 130 de la Bibliothèque d'Avranches ${ }^{22}$ : ms du Mont-Saint-Michel. Ce manuscrit a été écrit dans la seconde moitié du XIII ${ }^{\mathrm{e}}$ siècle, probablement au Mont-Saint-Michel où il semble être resté jusqu'au XV $\mathrm{XV}^{\mathrm{e}}$ siècle (comme le suggère une note du folio 1 : Ex libris S. Michaelis in periculo maris). Son titre est le suivant: Gesta Francorum a morte Caroli Calvi, id est ab anno dominice incarnacionis optingentesimo septuagesimo septimo usque ad annum nongentesimum sexagesimum sextum annum ejusdem dominice incarnationis.

Philippe Lauer indique que ces trois manuscrits dérivent d'un ancêtre commun, sans eux-mêmes dériver les uns des autres. Toutefois, E pourrait avoir été constitué à partir de deux manuscrits, dont $\mathrm{C}$, ou d'un manuscrit intermédiaire disparu. À présent que nous connaissons mieux les manuscrits de «la branche normande», voyons ce que nous pouvons en tirer.

\section{Reconstitution du manuscrit primitif de la branche normande}

Le but est d'arriver à caractériser le manuscrit $\beta$, ancêtre de la branche normande, en fonction de ses descendants C, D et E.

Essayons tout d'abord de le dater. Comme son ancêtre $\alpha$, son cousin B et ses descendants, il se termine obligatoirement par des notes relatives aux années 966978 : il ne peut donc pas être antérieur à cette dernière date. D'autre part, le plus ancien manuscrit descendant de $\beta$ est $\mathrm{C}$ et a été écrit au début du XI $\mathrm{XI}^{\mathrm{e}}$ siècle. Nous pouvons ainsi dater approximativement le manuscrit $\beta$ entre la fin du $\mathrm{X}^{\mathrm{e}}$ et le début du XI ${ }^{\mathrm{e}}$ siècle.

Analysons à présent les titres. Le point commun entre les deux plus anciens manuscrits $\mathrm{C}$ et D (XI ${ }^{\mathrm{e}}$ siècle) est le titre initial Gesta Normannorum.

Comme information supplémentaire, D ajoute cronica Frodoardi, presbiteri. Le même texte se retrouve, nous l'avons vu, à la fin des manuscrits, à l'année 966 . Il est fort probable que le copiste se soit inspiré de ce texte pour compléter son titre en indiquant le nom de l'auteur de l'œuvre. On notera qu'une personne a eu le même

21. FlodoARD, p. XLII-XLIII (ms D).

22. FlodoARd, p. XLIII-XLIV (ms E). Les Annales étaient visiblement inconnues du chroniqueur Robert de Torigni, abbé du Mont-Saint-Michel dans la seconde moitié du XII siècle. La première copie est donc probablement arrivée au Mont au plus tôt au XIII ${ }^{\mathrm{e}}$ siècle et il s'agit très certainement de ce ms E. 
comportement sur le manuscrit C, mais bien plus tard: le texte Chronica Flodoardi Presbyteri a été ajouté ultérieurement au-dessus du titre pour le compléter dans un objectif similaire ${ }^{23}$.

Le titre de E, plus récent de 200 ans, signale des Gesta Francorum et non pas Normannorum. Il précise la période couverte par ces gesta, en indiquant qu'elles débutent au décès de Charles le Chauve, en 877, et qu'elles s'achèvent en 966. Cette fois, le copiste a complété son titre grâce à des informations récoltées au début et à la fin des Annales. Le manuscrit E a dû être copié, du moins en partie, sur un autre dont le titre avait été corrigé : remplacement de Normannorum par Francorum comme sur C. À moins que la correction ait été effectuée par le copiste lui-même, qui désirait apporter à son manuscrit un titre correspondant davantage à son contenu.

Le titre de $\beta$ nous est donc donné par les deux plus anciens manuscrits dérivant directement de celui-ci et était probablement Gesta Normannorum. Ainsi, le titre initial du manuscrit $\mathrm{C}$ semble avoir été repris tel quel par son copiste.

Maintenant que $\beta$ a été plus précisément défini, voyons s'il est possible d'émettre une hypothèse sur l'identification de son auteur.

\section{Hypothèse d'identification du copiste du manuscrit primitif de « la branche normande »}

Le titre est particulièrement intéressant, surtout en le combinant avec la date de composition du manuscrit : celle-ci correspond précisément à la période où Dudon de Saint-Quentin a réalisé son histoire des premiers ducs de Normandie. Le titre de son œuvre De moribus et actis primorum Normanniae ducum a été imposé par André Duchesne puis repris par Jules Lair, les deux premiers éditeurs de Dudon ${ }^{24}$. Or, comme l'a souligné Gerda Huisman ${ }^{25}$ et comme l'a rappelé récemment Pierre Bouet ${ }^{26}$, «il conviendrait mieux de retenir l'intitulé Gesta Normannorum, voire Historia Normannorum, puisque ce sont ces dénominations que l'on rencontre sur de nombreux manuscrits ».

Il est intéressant de constater un titre similaire entre le manuscrit $\beta$ et certains manuscrits de Dudon, ce qui n'est probablement pas une coïncidence. Celui-ci auraitil pu amener lui-même le premier manuscrit des Annales de Flodoard en Normandie? Ce titre était visiblement présent sur le plus ancien manuscrit de « la branche normande» des Annales (ms $\beta$ ), alors que les autres manuscrits anciens n'appartenant

23. FlODOARD, p. LXX (fac-similé C). LAUER, 1898, planche XIII (fac-similé).

24. DuCHESNe, 1619, p. 49. DUdON DE SAINT-QuENTIN, p. 115. On notera qu'André Duschesne et Jules Lair ont peut-être repris le titre du manuscrit de Saint-Évroult, sur lequel ils se sont probablement basés en partie pour leur étude, et qui a aujourd'hui disparu: c'est peut-être le copiste de Saint-Évroult - et non le premier de ces deux éditeurs - qui s’inspira de la préface dédicatoire de l'œuvre pour donner un titre à son manuscrit ou pour compléter celui-ci jugé incomplet.

25. HUISMAN, 1983, p. 122-135.

26. BOUET, 2002, p. 58. 
pas à cette branche (ms A et $\mathrm{B}, \mathrm{XI}^{\mathrm{e}}$ siècle) ne comportent pas de titre ${ }^{27}$. Qu'un auteur, chargé d'écrire une histoire de Normandie, ait apporté à Fécamp une copie des Annales de Flodoard, sous le titre de Gesta Normannorum, afin de l'utiliser comme source pour constituer son œuvre, semble une hypothèse fort plausible. Davantage, en tous les cas, que de voir Guillaume de Volpiano arriver au même endroit, avec cette même copie, sous ce même titre, dans le but de réformer l'abbaye de Fécamp comme nous l'avons vu précédemment ${ }^{28}$. Dudon, contrairement à l'abbé de Dijon, avait un mobile pour agir de la sorte: il était chargé par le duc de Normandie d'écrire des Gesta Normannorum ${ }^{29}$, et ces Annales tiennent une place importante dans la rédaction de son œuvre.

On peut toutefois s'interroger sur les motifs ayant poussé Dudon à choisir ce titre pour sa copie des Annales: cet ouvrage est en effet tout sauf un traité d'histoire normande ${ }^{30}$ ! Une telle rubrique, qui ne correspond pas à son contenu, est étonnante, mais n'a sans doute rien d'anodin. Parmi les hypothèses imaginables pour justifier ce choix, nous en retiendrons trois, très différentes:

Première hypothèse : il est possible que Dudon ait d'abord récolté un certain nombre de documents avant de se lancer dans la rédaction de son ouvrage; ne connaissant ni la Normandie, ni l'histoire de son peuple, il lui aurait été difficile de commencer aussitôt sa rédaction sans disposer d'un minimum de sources à leur sujet. Il aurait alors pu les regrouper ensemble, afin de les exploiter plus aisément par la suite. Le dossier ${ }^{31}$ ainsi constitué pouvait très bien avoir reçu en tête les Annales de Flodoard, puisque ces dernières lui permirent de constituer l'épine dorsale de son œuvre $^{32}$. Si Dudon voulait donner un titre à ce dossier pour le caractériser, Gesta Normannorum semblerait tout à fait adéquat: le point commun des différents ma-

27. Le manuscrit original de Flodoard ne devait pas porter de titre, comme c'était souvent l'usage à l'époque. Richer (tome I, Prologue, p. 4-5), qui utilise les Annales pour rédiger son œuvre, les désigne dans sa préface dédicatoire simplement sous le terme Libellus ( Flodardi Presbyteri Remensis Libello »), ce qui reste plutôt vague, et conforte cette opinion. Le ms B n'ayant pas de titre, il y a de fortes probabilités pour que son ancêtre $\alpha$ n'en comporte pas non plus. Le ms $\beta$ fut donc vraisemblablement la plus ancienne copie des Annales de Flodoard portant un titre, et celui-ci - Gesta Normannorum - est loin d'être celui qui décrit le mieux son contenu.

28. Ce titre peut expliquer le fait que Guillaume de Volpiano, chargé de la réforme du monastère de Fécamp, ne prêta jamais attention à cette œuvre pourtant présente dans cette abbaye dès la fin du $\mathrm{X}^{\mathrm{e}}$ siècle : il ne se douta probablement jamais que derrière ces « actes des normands » se cachait en réalité des annales loin d'être restreintes à cette région. De plus, il connaissait sûrement sous ce même titre l'œuvre de Dudon, dont le style rhétorique, en tant que clunisien, devait le rebuter!

29. DudOn DE SAINT-QUentin, p. 119 et suiv. ; LifSHITZ, Felice, préface, chap. 1.

30. C'est malgré tout une source capitale pour connaître certains événements de l'histoire de Normandie au $\mathrm{X}^{\mathrm{e}}$ siècle.

31. Les recueils de manuscrits regroupant un même thème n'étaient pas rares aux $\mathrm{X}^{\mathrm{e}}$ et $\mathrm{XI}^{\mathrm{e}}$ siècles. Les deux plus anciens manuscrits des Annales de Saint-Bertin nous sont précisément parvenus dans des recueils de textes historiques constitués à ces époques.

32. Henri Prentout (1916, p. 26-27) indique que les Annales constituent la base principale de l'œuvre de Dudon pour la narration des événements de la période 919-966 relatifs à Rollon (livre II), Guillaume Longue-Épée et Richard I ${ }^{\text {er }}$ (livre III). 
nuscrits le constituant serait précisément de rapporter des faits relatifs aux actes des Normands ${ }^{33}$. Que l'ensemble des sources soit regroupé ainsi sous le nom du futur titre de l'œuvre de Dudon semble une théorie envisageable, bien qu'elle soit en réalité assez peu probable. En effet, elle suppose l'existence assez surprenante d'un manuscrit hétérogène ayant un titre général.

Seconde hypothèse : régulièrement présent à la cour fécampoise de Richard I ${ }^{\text {r }}$ à partir de 987, Dudon aurait très bien pu entendre parler du dessein de ce dernier, soucieux de disposer d'une œuvre sur ses ancêtres, depuis leur établissement en Normandie. S'il connaissait les Annales de Flodoard à cette époque, notre chanoine savait pertinemment qu'elles relataient nombre d'événements et de faits relatifs aux Normands. Désirant attirer l'attention du duc, il aurait très bien pu lui offrir une copie de cette œuvre sous le titre abusif de Gesta Normannorum ${ }^{34}$. Le duc, ravi, aurait alors sollicité Dudon pour la rédaction d'une historiographie répondant à l'image politique voulue par les Normands à la fin du $\mathrm{X}^{\mathrm{e}}$ siècle. Si cette hypothèse n'a rien d'impossible, elle reste malgré tout relativement improbable, puisque Dudon ne présente pas les choses ainsi dans sa préface dédicatoire.

Troisième hypothèse : le chanoine de Saint-Quentin aurait-il pu inscrire la note «Gesta Normannorum » en tête du ms $\beta$ des Annales, afin de caractériser son manuscrit en fonction de l'intérêt qu'il lui portait? Cette annotation serait donc ni une glose marginale ou interlinéaire destinée à informer les lecteurs ultérieurs, ni un titre destiné à indiquer le contenu exact de l'œuvre, mais une simple note personnelle permettant à notre auteur de visualiser rapidement le rôle que devrait jouer ces Annales dans la constitution de son histoire. En consultant ses différents manuscrits, Dudon pouvait instantanément identifier celui-ci comme devant lui fournir les informations relatives aux hauts faits des Normands. Cette note serait devenue accidentellement un titre dans les copies ultérieures : les scribes des manuscrits $C$ et D auraient mal interprété cette phrase précédant le début des Annales - qui ne leur était pas

33. Parmi les autres œuvres mentionnant des actes des Normands utilisées par Dudon, Henri Prentout $(1916$, p. 27) indique certaines annales carolingiennes et germaniques pour la constitution du premier livre: les Annales de Saint-Bertin, les Annales de Saint-Vaast, la Chronique de Réginon de Prüm, les Annales de Fulda, etc. Toutefois, seule l'utilisation des Annales de Saint-Bertin semble relativement évidente. La division géographique du monde s'inspire du Getica ou De Getorum sive Gothorum origine et rebus gestis de Jordanès (p. 34-35) et des Histoires contre les païens de Paul Orose (p. 34). L'Historia Langobardorum de Paul Diacre (p. 39) sert à Dudon pour justifier les invasions scandinaves. Pour les autres livres, Henri Prentout mentionne également La Complainte Latine relative à l'assassinat de Guillaume Longue-Épée (p. 31). Si l'on suit l'hypothèse de Jacques Le Maho (2001, p. 32) concernant l'existence d'une Vita Guillemi disparue d'où fut tirée cette fameuse complainte, nous pouvons suggérer que Dudon a également pu l'utiliser pour rédiger son texte relatif à Guillaume Longue-Épée. Leah Shopkow (1989, p. 34, n. 1) envisage également la possibilité de la connaissance par Dudon de l'Historia Francorum Senonensis, mais émet quelques réserves.

34. Albert de Vermandois étant mort en 987, Dudon n'avait dès lors plus de protecteur : cette démarche aurait pu viser à obtenir la protection du duc Richard. 
destinée - et en aurait fait le titre de leur copie, créant un décalage avec le contenu réel de l'œuvre.

Il est en réalité difficile de privilégier l'une ou l'autre de ces hypothèses: toutes semblent concevables et pourraient justifier du choix d'un tel titre. Mais aucune ne semble totalement satisfaisante, à l'exception peut-être de la dernière. En fait, l'explication est peut-être toute autre, et la question sur le sujet reste totalement ouverte...

Mais dans l'hypothèse où Dudon a bien introduit les Annales de Flodoard en Normandie, où a-t-il pu copier ce manuscrit? Nous savons que $\beta$, tout comme $B$, est issu d' $\alpha$, ancêtre du second groupe défini par Philippe Lauer. Or ce manuscrit est l'œuvre du continuateur de Flodoard. C'est ce manuscrit disparu qu'il va désormais falloir étudier au travers de ses différents descendants connus.

\section{Étude des notes « du continuateur» des Annales de Flodoard}

Les manuscrits B, C, D, E, F et G ont tous la particularité d'appartenir au groupe issu d'une copie des Annales de Flodoard ayant reçu les additions d'un continuateur. En effet, des notes postérieures à la mort de Flodoard ont été ajoutées sur les années 966, 976, 977 et 978. Ces additions ne figurent pas sur le manuscrit A, qui traite uniquement de la période 919 à $966^{35}$. Ainsi, les notes portant sur l'année 877 - absentes sur le ms A, mais ajoutées en tête des autres manuscrits - sont probablement elles aussi des ajouts de ce continuateur. Ce sont ces additions, portant sur ces cinq années, que nous allons à présent étudier. Elles peuvent être résumées et analysées ainsi ${ }^{36}$ :

- Année 877 : il s'agit d'un obit sur l'empereur Charles - mort le 7 octobre - fils de l'auguste empereur Louis et petit-fils de l'empereur Charles le Grand, digne représentant de César. Il est aussi question de l'abbesse Bertrade, sa noble parente, qui prie pour lui et a fait rédiger un abrégé de sa vie à sa mémoire.

Philippe Lauer pense que ces informations ont été tirées d'un obituaire de l'abbaye royale de Faremoutiers, où Bertrade, nièce de Charles le Chauve, fut abbesse $^{37}$. On remarque que les rois carolingiens mentionnés (Charles II le Chauve, Louis Ir le Pieux et Charlemagne) sont uniquement des rois ayant porté le titre impérial sur tout ou partie des territoires francs constitués par Charlemagne. Charles le Chauve fut d'ailleurs l'unique roi carolingien « originaire» de Francie occidentale à porter ce titre. Il n'y a aucune mention sur les

35. Le ms A comporte deux additions que l'on ne retrouve pas dans les autres: un texte hagiographique relatant les Visions de Flothilde figure avant les Annales (FLODOARD, p. 168-176). Une lettre d'un comte Renaud adressée à un duc G. d'Aquitaine, a été recopiée à la suite de l'année 966 . Elle contient une généalogie des descendants de Gerberge de Saxe, sœur d'Otton Irer, qui épousa Gilbert de Lorraine (mort en 939), puis se maria avec le roi de France Louis IV d'Outremer en secondes noces (FLODOARD, p. XXXIV et p. 159).

36. Pour ces cinq paragraphes et leur analyse, voyez surtout FLODOARD, p. 160-164 et 167; LOT, 1891, p. $72-73$ et $78-90$.

37. LAUER, 1900, p. 259-260; FlODOARD, p. 167. On peut aussi supposer que le continuateur connaissait l’abrégé de la vie de Charles le Chauve que fit rédiger Bertrade. 
autres rois qui se sont succédé à leur suite entre 877 et 919, à savoir Louis II le Bègue, Louis III, Carloman, Charles le Gros, Eudes et Charles III le Simple. C'est donc le titre d'empereur qui intéresse ici le continuateur des Annales: ces derniers, carolingiens, sont rapprochés des empereurs romains Auguste et César.

- Année 966: il s'agit d'un obit sur Flodoard, prêtre de l'Église de Reims et auteur de ces Annales, mort le 28 avril. Le continuateur fait également allusion aux autres œuvres littéraires de Flodoard, dont il vante les qualités. Suit la mention d'un énorme orage qui est à l'origine d'une gigantesque inondation le 23 juillet. Le continuateur donne simplement ici des informations sur l'auteur des Annales. Il tire probablement une partie de ses renseignements du nécrologe de l’Église de Reims, mais il connaît suffisamment Flodoard pour mentionner l'existence de ses autres œuvres, auxquelles il fait allusion ici.

Quant au phénomène météorologique décrit avec ses conséquences, il est daté avec précision et sous-entend la présence du continuateur des Annales à Reims à cette époque, ainsi que la rédaction de cette addition peu de temps après les faits.

- Suit une succession d'années sans commentaire de 967 à 975.

- Année 976 : il est question des travaux effectués par l'archevêque Adalbéron de Reims dans l'église Sainte-Marie, qui ne sont visiblement pas du goût du continuateur des Annales: il indique qu'Adalbéron est archevêque plus de nom que de mérite! On voit ici clairement la colère du continuateur, qui assiste à ce qu'il considère comme un véritable sacrilège: détruire ainsi d'anciens édifices religieux, même pour en reconstruire de nouveaux, n'est pas une action digne d'un archevêque, selon lui. On apprend cette même année (le 19 avril) une bataille opposant Charles aux comtes lorrains Godefroi et Arnoul. Les pertes sont importantes de part et d'autre. Godefroi lui-même tombe à terre percé d'une lance et gravement blessé : retrouvé par les siens au soir de la bataille, il survit à ses blessures, mais ne recouvre pas totalement la santé. Quant à Arnoul, il semble qu'il se soit enfui jusque dans ses domaines.

La bataille, dont il est question ici, oppose Charles de Lorraine - fils de Louis IV d'Outremer et frère du roi Lothaire - à Godefroi le Captif, comte de Methingau, de Hainaut et de Verdun, et à Arnoul, fils du comte de Cambrai et de Valenciennes. Charles avait rejoint le parti de Rénier et de Lambert, fils du comte Rénier III de Hainaut. Ceux-ci assiégèrent la ville de Mons, dans l'espoir de reprendre possession du Hainaut, dont Rénier III avait été dépossédé par l'empereur Otton Ir $^{\text {er }}$ et son frère l'archevêque Brunon de Cologne en 957. Ces comtés avaient été confiés en 974 à Arnoul et à Godefroi ${ }^{38}$. Le sort de ce dernier est décrit en détail et semble intéresser particulièrement le continuateur des Annales. Le fait qu'il est renseigné sur sa santé longtemps après le siège de Mons laisse supposer une certaine proximité avec celui-ci, mais également que ces

38. Sur les événements relatifs au Hainaut entre 957 et 977, voyez SASSIER, 1987, p. 142, 160-161 et 162. 
lignes ont été rédigées quelque temps après les faits. La fuite d'Arnoul et sa passivité suite à son retour dans ses terres semblent plus ou moins directement critiquées. On notera que Rénier et Lambert, principaux protagonistes, ne sont pas mentionnés par le continuateur : ces personnages ne l'intéressent visiblement pas.

Enfin, il est question du décès de l'évêque de Laon, Roricon (949 - 976), à la suite d'une longue paralysie.

Cette information, concernant l'évêché de Laon, est en fait à relier directement à toutes celles données au sujet de son successeur, l'année suivante.

- Année 977 : il s'agit d'une description très détaillée de l'accession d'Adalbéron le jeune à l'évêché de Laon (sont décrits: le don de l'évêché par le roi carolingien Lothaire le 16 janvier à Laon; l'ordination comme prêtre le 24 mars par l'archevêque de Reims Adalbéron; le sacrement à Reims le $1^{\text {er }}$ avril; la consécration de l'évêque à Laon et l'établissement sur le siège épiscopal le jour de Pâques, c'est-à-dire le 8 avril).

Il est aussi question du décès de l'évêque Hadulfe de Noyon à la suite d'une longue paralysie.

Ce sont les informations concernant Adalbéron de Laon ${ }^{39}$, qui prédominent sur l'ensemble des événements de l'année. Adalbéron se voit attribuer le terme juvenis probablement dans le but d'être distingué de son homonyme l'archevêque de Reims, dont il a été fait mention précédemment, et qui appartient comme lui à la «Maison d'Ardenne». Il était chancelier du roi Lothaire depuis 974 et, par conséquent, était «jeune» mais sans doute déjà adulte à cette épo$q^{40}{ }^{40}$. L'auteur aurait pu désigner le nouvel évêque de Laon par Ascelin, le second nom sous lequel il est également connu, pour lever l'ambiguïté entre l'oncle et le neveu homonyme. On peut supposer soit qu'il n'a pas voulu l'employer, soit que le nom d'Ascelin ne lui était pas encore attribué alors. Quoi qu'il en soit, Adalbéron intéresse incontestablement le continuateur des Annales: toutes les étapes de son accession au siège épiscopal sont décrites dans les moindres détails, alors que cela tient généralement dans les annales classiques en une simple ligne. On notera aussi le fait qu'il s'agit du seul évêque pour lequel l'accession est mentionnée entre 976 et 978 : on sait pourtant qu'il y en eut plusieurs autres sur cette période ${ }^{41}$. D'ailleurs, le continuateur mentionne

39. Sur l'évêque Adalbéron de Laon, voyez ADALBÉron DE LAON, p. IX-XVIII et CoOLIDGE, 1965, p. 1 et suiv.

40. Voyez le terme juventus, étymologiquement proche de juvenis (LETT, Didier, "Âges de la vie», in Dictionnaire du Moyen-Âge, p. 16). Ce terme désigne généralement, à l'époque, des hommes âgés de 21 à 35 ans. On notera qu'un siècle plus tôt l'évêque Hincmar de Laon était appelé «le jeune » pour le distinguer de son oncle l'archevêque Hincmar de Reims: le procédé est ici probablement identique. Par ailleurs, le terme juvenis peut également prendre le sens de "célibataire », et donc être indépendant de l'âge de l'individu.

41. Sur les successions d'évêques entre 975 et 978, voyez LOT, Ferdinand, 1891, p. 89-90. En plus de Laon, Ferdinand Lot mentionne cinq autres évêchés : Amiens, Sens, Autun, Soissons et Noyon. 
le décès de l'évêque de Noyon cette même année, sans parler de son successeur par la suite. Cet évêque de Noyon, Hadulfe (955 - 977) avait participé aux côtés de Roricon de Laon à la consécration de l'archevêque de Reims Odelric - parent de la famille d'Ardenne - en 962.

- Année 978: la note porte uniquement sur le décès de l'abbé Mac Allan de Saint-Michel-Archange (Saint-Michel-en-Tiérache), le jour de la saint Vincent (22 janvier ou 9 juin).

Originaire d'Irlande, il avait été choisi par l'évêque de Laon Roricon comme abbé du monastère Saint-Vincent-de-Laon lors de la réforme de 961, au cours de laquelle douze moines de Saint-Benoît-sur-Loire (Fleury) vinrent remplacer les chanoines.

Après nous être intéressé au fond, attardons-nous un instant sur la forme, afin de vérifier l'homogénéité du style du continuateur. En fait, deux années se démarquent nettement des autres : il s'agit des années 877 et 966, qui comportent un certain nombre de similitudes présentes ni chez Flodoard, ni dans les autres additions. En effet, ces deux paragraphes, comportant les obits de Charles le Chauve et de Flodoard, sont construits de façon identique : ce sont des éloges, un peu pompeux, où l'auteur emploie de nombreux superlatifs pour évoquer les abondantes qualités des défunts, dans un style littéraire relativement travaillé. Ce style tranche nettement avec celui plus sobre et plus neutre utilisé par Flodoard et même par le continuateur dans les autres additions ${ }^{42}$. D'autre part, la formulation de la phrase indiquant l'espoir de l'accès des défunts au Royaume des Cieux est particulièrement intéressante: dans les deux cas, on retrouve la proposition caractéristique «ut credimus », que l'on ne retrouve nulle part ailleurs dans l'ensemble des Annales. Nous avons donc probablement affaire à deux continuateurs distincts : un premier pour les années 877 et 966 ; un second pour les années 976 à 978.

Cette constatation se confirme lors de l'analyse détaillée des dates. La précision de la datation des événements mentionnés dans ces additions est remarquable: presque tous les faits décrits entre 966 et 978 sont datés au jour près, de la mort de Flodoard à celle de l'abbé Mac Allan, en passant par les inondations, la bataille de Mons, les différents événements intervenant dans la succession des évêques de Laon et le jour du décès d'Hadulfe de Noyon ${ }^{43}$. Il semble donc évident que les faits ont été rapportés très peu de temps après qu'ils se soient produits. La mention du prix du muid de vin pour les années 976 et 977 va également dans ce sens. Or, il existe une

42. Flodoard et le continuateur des Annales sur la période 976-978 mentionnent des décès d'hommes importants sans avoir recours une seule fois à la rédaction de tels obits, en total décalage avec le style habituel employé dans des annales. Le continuateur des années 976-978 préfere narrer l'état physique ou psychologique des défunts peu avant leur mort, plutôt que de se lancer dans un panégyrique ou une succession d'éloges.

43. Flodoard ne s'est malheureusement pas montré aussi précis pour la plupart des événements décrits sur la période 919-966: des dates précises sont fournies pour les phénomènes météorologiques et les miracles, alors que leur absence se fait souvent cruellement ressentir pour les faits historiques, tels que les batailles ou les traités (FLODOARD, p. XVII). 
longue interruption de près de dix années entre 966 et 976. A priori, on pourrait penser qu'un continuateur a pu rédiger ses additions à partir de cette dernière date, et ajouter des informations sur les années 877 et 966 à cette époque. Mais la datation extrêmement précise des inondations survenues en 966 exclut cette hypothèse: comment un auteur pourrait restituer aussi fidèlement la date du 23 juillet en écrivant une dizaine d'années après les faits? On s'attendrait davantage à une information un peu plus vague, comme «durant l'été 966 » ou, s'il a un excellent souvenir du phénomène, «courant juillet 966 ». Avec une telle mémoire, il aurait très certainement été capable de mentionner d'autres événements advenus entre 967 et 975, comme par exemple ceux survenus dans le Hainaut en 973-974, ce qu'il n'a pas fait. Les événements rapportés en 966 sont donc contemporains de leur rédaction, qui a vraisemblablement eu lieu cette même année.

Ainsi, l'analyse de la forme et la datation des événements nous permettent d'établir une intervention de deux continuateurs distincts pour rédiger ces cinq paragraphes. Le premier copie les Annales vers 966, peu après la mort de Flodoard et probablement au moment des inondations survenues en juillet. Il en profite pour ajouter deux paragraphes contenant essentiellement des obits dans un style littéraire travaillé : celui concernant l'année 877 est ajouté en tête des Annales; le second, concernant l'année 966, est ajouté à la fin. Le copiste réalise donc plutôt des additions qu'une véritable continuation de l'œuvre de Flodoard. Un second scribe prend la plume entre 976 et 978 pour rapporter des informations sur ces trois années en fin d'Annales : la démarche est bien ici celle d'un continuateur, qui respecte le style de l'œuvre en rapportant différents événements contemporains dans l'ordre chronologique.

Les événements mentionnés par les deux continuateurs des Annales nous renseignent sur l'espace, le temps et les individus connus d'eux. Il est possible de caractériser ces informations (voir tableau p 15-16) ${ }^{44}$.

Nous observons que la totalité des événements localisés sont situés dans quatre pagi de la province de Reims, c'est-à-dire qu'ils concernent des évêchés et des territoires relevant de cet archevêché ${ }^{45}$. À l'exception de la bataille de Mons, qui a lieu dans le Hainaut, tous sont situés en France, dans le royaume de Lothaire. Ils se déroulent en fait dans trois pagi voisins : le Rémois, le Laonnois et le Noyonnais. Mais on remarque surtout un glissement des lieux mentionnés au cours du temps : si les informations localisées concernent exclusivement Reims en 966 (soit $100 \%$ des informations sur cette période), ce sont celles relatives à l'Église de Laon qui prédominent ensuite à partir de 976 (45,4 \% des informations de la période 976-978, contre seulement 11,3\% pour Reims). À partir du 20 décembre 976, cet intérêt pour l'Église de Laon s'impose d'avantage (71\% des informations de cette période, contre 3,2\% seulement pour Reims). Le premier continuateur se trouvait donc vraisemblablement

44. Le nombre de lignes indiqué correspond à celui comptabilisé dans l'édition des Annales de Philippe Lauer.

45. Sur ces quatre pagi, voyez Sot, Michel, p. 30-32, et en particulier la carte 5, p. 30. 


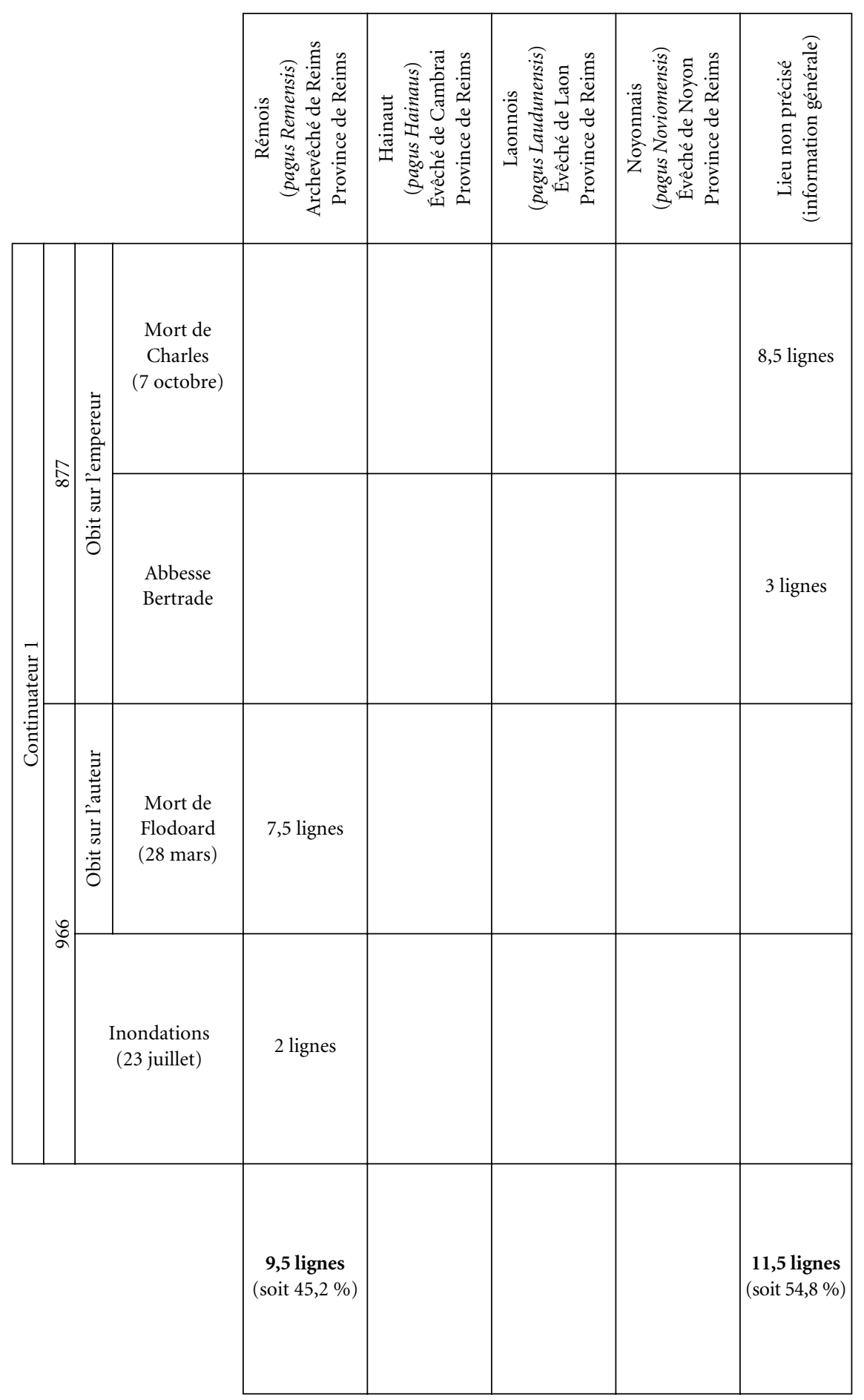

Tabularia «Études », n 4, 2004, p. 1-38, 15 janvier 2004 


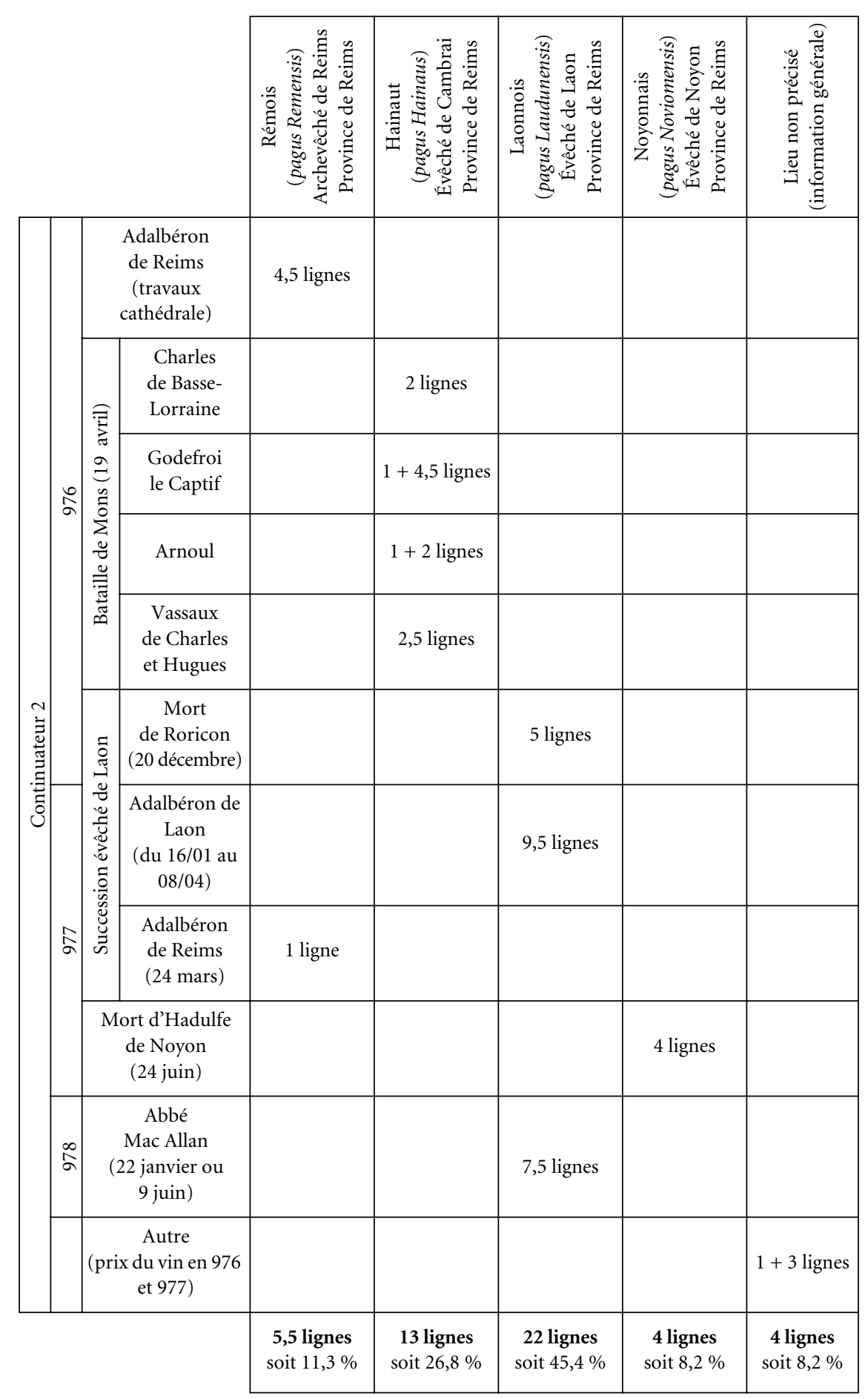


à Reims lorsqu'il copia les Annales en 966, ce qui semble assez logique, puisque l'original de Flodoard s'y trouvait. Mais le second continuateur était très certainement à Laon lorsqu'il fit les additions postérieures au 20 décembre 976 : ce scribe est sans doute un clerc de cette église.

En fait, il n'est pas surprenant de trouver trois des manuscrits les plus anciens des Annales de Flodoard à Reims (ms original), à Laon $(\mathrm{ms} \alpha)$ et à Soissons (ms B) : ces trois villes voisines étaient étroitement liées au $\mathrm{X}^{\mathrm{e}}$ et $\mathrm{XI}^{\mathrm{e}}$ siècles ${ }^{46}$. En revanche, il est plus étonnant d'en trouver d'autres à Fécamp et en Normandie à cette même époque ( $\mathrm{ms} \beta, \mathrm{C}$ et $\mathrm{D})$ : les liens géographiques et historiques de Reims avec cette ville et cette région sont, a priori, moins évidents à définir.

\section{Étude des additions du second continuateur (années 976 à 978)}

Dans ces additions, la prédominance des informations concernant l'Église de Laon n'est pas la seule remarquable: celle relative aux membres de la «Maison d'Ardenne », famille se rattachant à la branche carolingienne de Lotharingie (voir figure $1^{47}$ ), est elle aussi significative. Le patrimoine de cette puissante famille s'étendait sur une région comprise entre la Meuse à l'ouest, les Ardennes au nord, Trèves à l'est et les trois diocèses de Verdun, Toul et Metz au sud ${ }^{48}$. Les évêques de cette dernière ville, qui était alors la cité la plus puissante de Lorraine, furent d'ailleurs pour la plupart issus de cette famille du $\mathrm{X}^{\mathrm{e}}$ au $\mathrm{XI}^{\mathrm{e}}$ siècle ${ }^{49}$. Dans la seconde moitié

46. Géographiquement proches, la communication entre les villes de Reims, Laon et Soissons se faisait d'autant plus facilement que des voies romaines reliaient ces trois villes (SOT, Michel, carte 6, p. 40). On notera également la facilité de communication avec la ville de Saint-Quentin, elle aussi voisine : Laon était située à proximité de la voie romaine Reims/Saint-Quentin. Historiquement, le triangle Reims-Laon-Soissons apparaît à l'époque de saint Rémi, qui crée vers la fin du V $\mathrm{V}^{\mathrm{e}}$ siècle un siège épiscopal à Laon pour son neveu, dont le frère occupait déjà celui de Soissons (SOT, Michel, p. 699). Ce triangle relationnel fort apparaît de nouveau après 925, sous les épiscopats des archevêques Hugues de Vermandois et Artaud (SOT, Michel, p. 346, 706).

47. Cette reconstitution généalogique simplifiée a été faite à partir des sources suivantes: PARISSE, 1976, p. 19-22 et 844-849 (Annexe E); PARISSE, 1993, p. 89; POUll, 1991, p. 14 ; Poull, 1994, p. 7-16 et p. 66 ; ADALBÉRON DE LAON, 1979, p. IX-XI; PARISOT, 1909, p. 6 et suiv. (notamment p. 6-20); PARISOT, 1919, p. 189-191; Histoire du Luxembourg, 2002, p. 90-92; LE JAN, Régine, 1995, p. 37, 184, 190, 294-295, 321, 323, 407, 417-419, 456; FlODOARD, 1906, an. 951 (p. 130131), 954 (p. 139), 959 (p. 147) et an. 962 à 966 (p. 154-158); LOT, 1891, p. 13, p. 40 n 2, p. 50 $\mathrm{n}^{\circ} 1$, p. $55 \mathrm{n}^{\circ} 2$, p. $63-64$, p. $68 \mathrm{n}^{\circ} 2$, p. 80 , p. 141 , p. $149-150$, p. $158 \mathrm{n}^{\circ} 1$, p. $245 \mathrm{n}^{\circ} 4$, p. $287 \mathrm{n}^{\circ} 1$ et addition p. 409; GERBERT, 1889, p. 26-28.

48. Voyez PARISSE, 1976, carte p. 22 bis; Histoire du Luxembourg, 2002, p. 93 + carte p. 86. Cette puissante famille regroupait de hauts dignitaires laïcs et ecclésiastiques : comtes, ducs, abbés laïcs, évêques et archevêques (LE JAN, 1995, p. 417-419). Les Adalbéron étaient généralement destinés à des fonctions cléricales (Ibidem, p. 216-217; Histoire du Luxembourg, 2002, p. 92).

49. Parmi les évêques de Metz des $\mathrm{X}^{\mathrm{e}}$ et XI ${ }^{\mathrm{e}}$ siècles appartenant à la famille d'Ardenne, notons : Wigeric? (919-927), Adalbéron I Ir (929-962), Adalbéron II (984-1005), Thierry II (1006-1047), Adalbéron III (1047-1072). Seules les périodes 927-929 et 962-984 semblent leur avoir échappé entre 919 et 1072 ! Metz était alors une ville symbole, où la mémoire et la tradition carolingienne étaient fortement implantée (Histoire du Luxembourg, 2002, p. 80 et 82) : Saint-Arnoul, ancêtre de la dynastie, fut évêque de cette ville au début du VIIe siècle. 


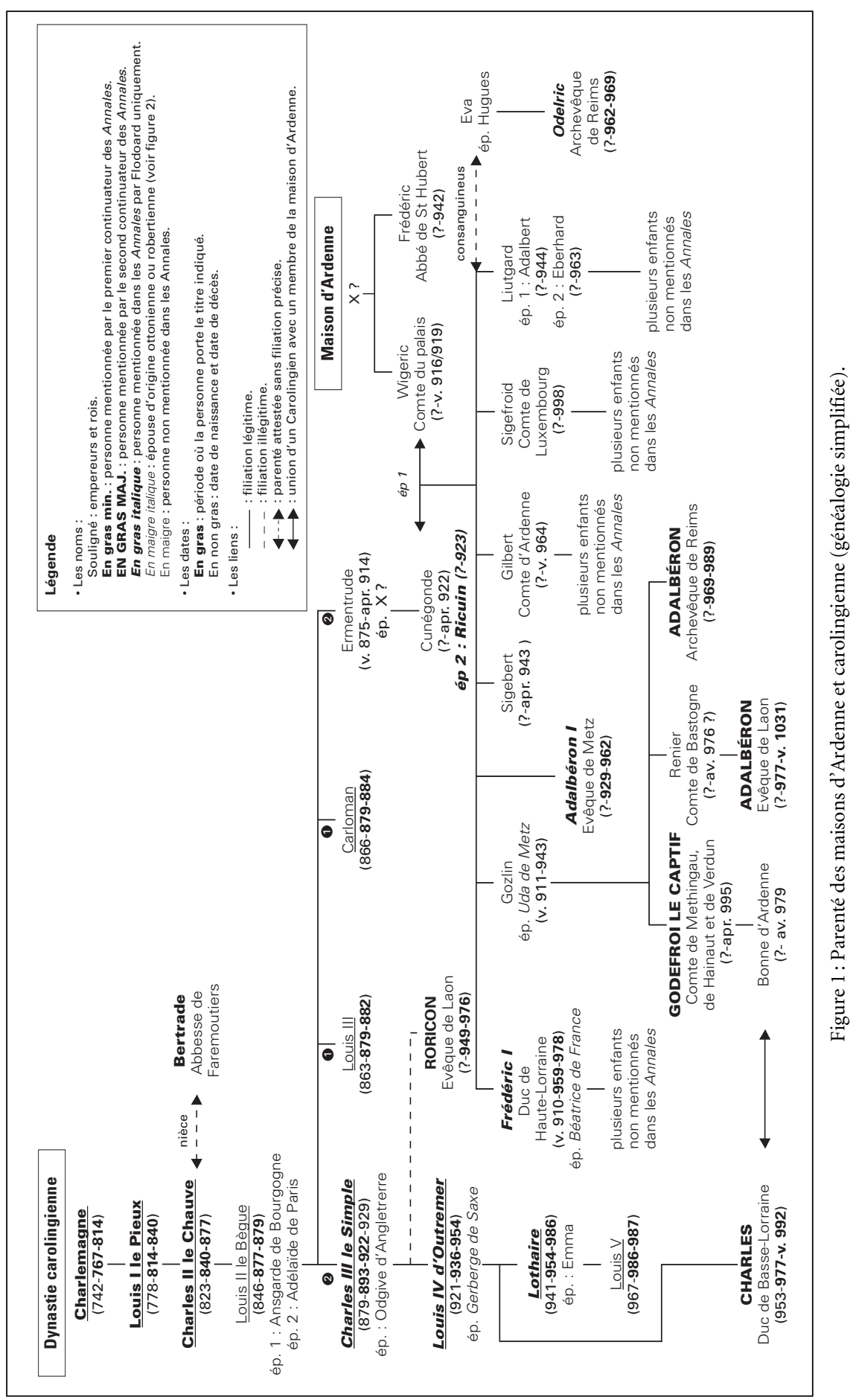


du $\mathrm{X}^{\mathrm{e}}$ siècle, leurs possessions s'étendirent dans le diocèse de Cambrai - entre Meuse et Escaut, avec le Hainaut et le Brabant - et avec deux évêchés en France, à Reims et à Laon ${ }^{50}$. Toutefois, c'est à une branche bien spécifique de cette Maison, divisée en plusieurs clans très liés, que le continuateur des Annales semble s'intéresser: seuls des descendants du comte Gozlin et de sa femme Uda sont mentionnés ${ }^{51}$ : Adalbéron de Reims ${ }^{52}$ (en 976 et en 977), Godefroi le Captif (en 976) et Adalbéron de Laon (en 977). Ajoutons également Charles de Basse-Lorraine, qui épousa une fille de Godefroi le Captif, et qui est lui aussi nommé en 976, lors de la bataille de Mons. À eux seuls, les deux oncles, leur(s) neveu (x) et/ou leur gendre occupent près de la moitié des informations relatives aux personnes citées sur cette époque $(46,4 \%)$. Le continuateur n'indique d'ailleurs aucun membre d'une autre branche de cette famille dans sa narration ${ }^{53}$. Ainsi, en excluant les faits concernant directement le Laonnois (succession à l'évêché et mort de Mac Allan), deux des trois autres événements mentionnés (travaux dans la cathédrale de Reims et bataille de Mons) concernent des membres de cette famille (Adalbéron de Reims, Godefroi le Captif et Charles de Basse-Lorraine). Outre le fait d'être proche de l'Église de Laon, on peut donc penser que le continuateur des Annales sur la période 976978 appartient à cette branche de la «Maison d'Ardenne» ou, du moins, entretient des rapports très étroits avec celle-ci.

Il est possible d'exclure immédiatement l'archevêque de Reims Adalbéron de la liste des continuateurs potentiels : il est vivement critiqué en 976 pour ses travaux de rénovation dans l'église cathédrale Sainte-Marie. On notera d'ailleurs au passage que le continuateur assiste lui-même à ces travaux, et donc qu'il se trouve à Reims à cette époque. Ensuite, à partir de décembre 976, les événements concernent avant

50. Le premier archevêque de Reims apparenté à cette famille fut Odelric (962-969), à qui succéda Adalbéron (969-989). Le neveu homonyme de ce dernier devint évêque de Laon de 977 à 1031. Au cours des $\mathrm{X}^{\mathrm{e}}$ et $\mathrm{XI}^{\mathrm{e}}$ siècles, la zone d'action de la "Maison d'Ardenne » s'étend de la frontière de l'Empire à l'Est, jusqu'au-delà de la Meuse à l'ouest et à la Flandre au nord. Ses membres occupent les évêchés de Metz, Reims, Laon et Verdun, et convoitent celui de Trèves; ils dirigent de nombreuses abbayes, dont celles de Gorze, Saint-Mihiel, Saint-Hubert, Stavelot-Malmédy, Echternach et Saint-Maximin de Trèves; leurs charges comtales s'exercent entre la Meuse et la Moselle, et ils se succèdent quasiment systématiquement à la tête des duchés de Basse et de Haute-Lorraine (Histoire du Luxembourg, p. 93 et carte p. 92).

51. C'est de cette branche que sont issus les comtes de Verdun (voyez PARISSE, 1993, p. 89; PARISSE, 1976, p. 846-847). Gozlin était carolingien par sa mère Cunégonde et sa grand-mère Ermentrude, fille de Louis II le Bègue. Uda était ottonienne par sa mère Uda de Saxe, fille d'Otton le Borgne et sœur d'Henri Irr l'Oiseleur (figure 1 et 2) : leurs enfants eurent donc une ascendance doublement royale (et pour ainsi dire doublement impériale).

52. Sur ce personnage, voyez SASSIER, 1987, p. 152-227 et 273-276 ; LOT, 1891, p. 64-65 ; «Adalbéron, archevêque de Reims ", in Histoire Littéraire de la France, t. VI, p. 444-450.

53. Il est remarquable de constater que le continuateur ne s'intéresse qu'à cette branche de la « Maison d'Ardenne». Aucune référence n'est faite à la branche des ducs de Lorraine et des comtes de Bar, issus du duc Frédéric Ir (au moins trois enfants: voyez PoULL, 1994, p. 7-66; PARISSE, 1976, p. 844-845) ou à celle des comtes du Luxembourg, issue du comte Sigefroid (au moins dix enfants: voyez Histoire du Luxembourg, p. 98-103; PARISSE, 1976, p. 848-849). Ces deux branches sont pourtant au moins aussi prestigieuses! 
tout le Laonnois: le décès de l'évêque Roricon, l'accession de son successeur Adalbéron et le décès de l'abbé Mac Allan, dont les monastères dépendaient de l'Église de Laon. La seule information ne concernant pas directement cette église sur cette période est relative à un évêché voisin, celui de Noyon.

À présent, voyons s'il est possible d'identifier l'auteur de ces additions à partir des renseignements à notre disposition.

\section{Proposition d'identification du continuateur des Annales de Flodoard sur la période 976-978}

Il existe au moins un représentant de la «Maison d'Ardenne» se trouvant à Reims avant 977 et à Laon ensuite: il s'agit de l'évêque Adalbéron de Laon, dont l'accession au siège épiscopal est mentionnée avec de très nombreux détails sur l'année 977. De fortes présomptions existent donc à son sujet pour le voir comme le continuateur de la période 976-978. Dans ce cas, il se donnerait une bonne place dans sa continuation des Annales, puisqu'une dizaine de lignes le concernerait personnellement. Or, cette caractéristique se retrouve dans deux des œuvres majeures d'Adalbéron: il apparaît nommément dans son De Summa Fidei, un dialogue le confrontant avec la Foi; de même, il s'identifie dans son Carmen ad Rotbertum regem, un autre dialogue l'opposant en personne au roi Robert le Pieux. L'évêque de Laon n'hésitait donc pas à se mettre en avant dans ses écrits ${ }^{54}$. On remarquera qu'il s'identifie lui-même par Adalbéron et non par Ascelin dans ces deux œuvres, de la même façon que le fait ici le continuateur des Annales ${ }^{55}$.

Mais Laon était déjà un centre scolaire réputé à cette époque, et Adalbéron fut loin d'être le seul érudit à s'y trouver aux alentours de 977. On peut donc se demander s'il fut directement le continuateur des Annales sur cette période ou si la rédaction de ces notices ne fut pas le fruit de l'un de ses élèves. Cette dernière hypothèse sousentendrait la présence de ce disciple aux côtés d'Adalbéron à Reims en 976, puis à Laon à partir de 977. En effet, nous avons vu que le continuateur avait personnellement assisté aux travaux ordonnés par l'archevêque de Reims dans l'église SainteMarie; de même, le continuateur aurait suivi l'évêque lors des différentes étapes l'ayant conduit au siège épiscopal, bien que celles-ci eurent lieu alternativement entre deux endroits: Reims et Laon. Il s'agirait donc d'un disciple particulièrement proche,

54. Faut-il y voir un certain égocentrisme chez cet évêque?

55. AdAlbÉRON DE LAON, p. XVIII-XXI. Certes, le style de la continuation des Annales est très différent de ceux employés dans les œuvres connues d'Adalbéron. Cependant, l'évêque de Laon a montré une grande aptitude à maîtriser tous les genres littéraires (ADALBÉRON DE LAON, p. XXXVIXXXVIII). D'autre part, il ne serait pas le premier auteur à avoir su varier les genres et les styles: rien qu'à Reims, Hincmar et Flodoard surent le faire avant lui, en réalisant des annales, des œuvres hagiographiques et des œuvres historiques, soit en vers, soit en prose, tout en sachant adapter leur style suivant le type d'ouvrage réalisé (SHOPKOW, 1989, p. 24, col. 2). 
accompagnant le jeune Adalbéron en toutes circonstances dès 976, alors que ce dernier n'était qu'un simple clerc. Un attachement aussi précoce semble assez surprenant. Cela sous-entendrait également un attrait tout particulier de ce disciple pour les faits et gestes des membres de la famille d'Ardenne proches d'Adalbéron, ainsi qu'une grande audace pour oser critiquer ouvertement les travaux architecturaux de l'archevêque, oncle de son maître. Ces deux derniers points paraissent pour le moins étonnants, à moins qu'Adalbéron de Laon lui-même ait guidé son élève dans la rédaction de ces quelques lignes ${ }^{56} \ldots$

A priori, notre évêque de Laon semble l’individu le mieux placé pour avoir rédigé ces additions: sa proximité avec les personnages et les lieux, ainsi que la concordance des dates mentionnées concourent à le désigner comme tel. Mais nous ne pouvons pas totalement écarter la possibilité que ce soit l'un de ses très proches disciples, clerc de l'Église de Laon, qui ait pu en être le rédacteur.

L'identification d'Adalbéron comme continuateur des Annales est d'autant plus séduisante qu'il existe de nombreux liens entre l'évêque de Laon et Dudon de SaintQuentin. Nous savons que le chanoine lui dédia son œuvre dans une préface élogieuse, qu'Adalbéron fut le correcteur de cette œuvre, et qu'il tint probablement une part déterminante dans la nomination de Dudon comme doyen de la collégiale de Saint-Quentin ${ }^{57}$. Nous avons déjà défini le mobile de Dudon pour l'apport d'une copie des Annales de Flodoard en Normandie et nous connaissons désormais le moyen à sa disposition pour y parvenir : son étroite relation avec l'évêque de Laon, continuateur probable des Annales de Flodoard. C'est en tout cas visiblement à Laon que Dudon put copier son manuscrit des Annales, qui devint par la suite le manuscrit primitif de «la branche normande » à Fécamp. L'origine laonnoise de ce manuscrit semble infirmer définitivement l'hypothèse de son introduction en Normandie par Guillaume de Volpiano : cette région ne fut nullement touchée par les réformes clunisiennes de l'abbé de Dijon qui, à notre connaissance, ne fut jamais en relation directe avec l'Église de Laon. De même, l'hypothèse de Philippe Lauer faisant de Brunon de Roucy, chanoine de Reims puis évêque de Langres (981-1015), l'auteur éventuel de ces additions semble elle aussi peu probable.

Reste à savoir comment Adalbéron de Laon ou son disciple s'est procuré cette version des Annales de Flodoard ayant déjà reçu des additions sur les années 877 et 966. L'a-t-il trouvée sur place à Laon lorsqu'il est devenu évêque, ou bien l'a-t-il

56. Voir Adalbéron critiquer son oncle, l'archevêque de Reims, de la sorte ne semble pas surprenant. L'évêque de Laon est célèbre pour ses confrontations avec les hommes de pouvoir de son époque, ainsi que ses prises de position vives : le comte Landri de Nevers (Rythmus satiricus), le roi Robert le Pieux et l'abbé Odilon de Cluny (Carmen ad Rotbertum regem) furent des cibles de choix dans ses œuvres. Il s'opposera ouvertement à plusieurs reprises à son ancien maître, Gerbert d'Aurillac, juste avant l'An mil (GERBERT, p. XXXII-XXXIII et appendice V p. 241). Ses confrontations avec son propre petit-cousin, Gérard, évêque de Cambrai (1012-1051) - petit-fils de Godefroi le Captif par sa fille Ermentrude - furent elles aussi virulentes (ADALBÉRON DE LAON, p. X-XI).

57. Dudon De SAInT-Quentin, Préface dédicatoire, p. 119 et suiv. Version électronique de Dudon par Felice Lifshitz, préface dédicatoire, chap. 1. 
apportée à cet endroit lui-même, alors qu'elle était auparavant à Reims ? Dans le premier cas, cela sous-entendrait une rédaction de l'année 976 postérieure d'un an aux événements, alors que le second cas pourrait indiquer une rédaction au fur et à mesure de 976 à 978. La narration des suites de la bataille de Mons indique clairement que l'écriture n'a pas eu lieu immédiatement après les faits : l'état de santé de Godefroi le Captif est mentionné longtemps après cette bataille, puisqu'il est indiqué que le comte survécut à ses blessures, mais ne recouvra jamais totalement sa première santé. Au moins plusieurs mois se sont donc écoulés entre les faits et la rédaction de ceux-ci. D'autre part, sur cette même année, la chronologie n'est pas totalement respectée : le décès de Roricon, survenu le 20 décembre, est mentionné avant les informations sur le prix du muid de vin au mois d'août. On peut donc privilégier la première hypothèse, à savoir une présence de cette copie des Annales à Laon avant l'arrivée d'Adalbéron en 977, et une rédaction de l'année précédente de façon rétrospective.

Suivant cette hypothèse, il convient à présent d'étudier les additions faites par le premier continuateur.

\section{Étude des additions du premier continuateur (années 877 et 966)}

Ce continuateur est avant tout un copiste des Annales de Flodoard, qui ajoute quelques informations complémentaires l'intéressant particulièrement. Ces dernières portent essentiellement sur les empereurs carolingiens et sur l'auteur des Annales. Dans le premier cas, l'ajout est fait au début de l'œuvre et dans le second cas, à la fin: on peut ainsi parler davantage d'additions que de véritables continuations.

Flodoard a eu recours à des copistes pour rédiger son Histoire de L'Église de Reims $^{58}$. En fut-il de même pour la rédaction de ses Annales, en particulier sur la fin de sa vie, où il était visiblement diminué au point de renoncer aux charges de son ministère ${ }^{59}$ ? Ainsi, l'un des copistes ayant travaillé pour Flodoard pourrait être l'auteur des additions portant sur les années 877 et 966 . L'hypothèse, si elle est séduisante, laisse cependant planer quelques doutes: pourquoi ne pas avoir fait ces additions directement sur l'œuvre originale de Flodoard? En effet, puisqu'elles n'apparaissent pas sur le manuscrit A de Dijon, pourtant complet sur la période 919-966, on peut en déduire que c'est bien sur une copie et non sur l'original qu'elles ont été apportées. Quel intérêt aurait eu ce continuateur de copier les Annales si son intention était de les laisser à Reims ? L'église disposait déjà de la version originale, et on avait peu coutume, à l'époque, de conserver des œuvres en plusieurs exemplaires dans les archives et les bibliothèques. La copie d'un manuscrit se traduisait généralement par le désir de le diffuser en un autre lieu. À moins que la motivation

58. SOT, 1993, p. 103.

59. FlODOARD, an. 963 (p. 154). Flodoard renonce à son ministère et à ses charges en 963, soit trois ans avant son décès («ministerium abdicavi pralaturae»). 
du copiste a été de mettre au propre l'original des Annales, considéré comme un brouillon, nous ne comprendrions pas une telle démarche. Mais dans ce cas, les informations relatives aux années 877 et 966 paraissent plutôt suspectes si elles émanent d'un simple copiste; en effet, le scribe ne s'est pas contenté de reproduire exactement l'original, mais il a ajouté des informations très précises. Où trouva-t-il ces informations, notamment au sujet de Charles le Chauve et de sa nièce Bertrade, et dans quel but les ajouta-t-il en tête des Annales? Par ailleurs, l'emploi d'un style littéraire travaillé va là encore à l'encontre de l'hypothèse du scribe simple copiste.

En fait, Flodoard semble avoir eu recours à des scribes, non pas pour rédiger ses manuscrits originaux, mais plutôt pour les copier afin de les transmettre à d'autres personnes: ce fut le cas du manuscrit de son Histoire de l'Église de Reims, envoyé " au prélat R. », à qui l'œuvre fut dédiée. D'ailleurs, une lecture attentive des Annales permet de constater la diminution des facultés du chanoine dans ses dernières années: les paragraphes deviennent plus courts, les informations sont plus concises, et parfois même redondantes. Flodoard a donc bien rédigé lui-même ses Annales jusqu'au bout, sans avoir recours au moindre scribe, malgré les infirmités causées par sa vieillesse ${ }^{60}$.

L'auteur de ces additions nous donne cependant indirectement des renseignements intéressants à son sujet. Il connaissait visiblement bien l'œuvre de Flodoard: son allusion à la qualité de ses autres livres le montre suffisamment, et ce n'est pas dans le nécrologe de Reims qu'il put trouver ces informations. Nous n'avons donc pas affaire à un simple copiste, mais à un érudit connaissant les autres œuvres majeures du chanoine de Reims, à savoir De triumphis Christi et Historia Remensis $E_{c c l e s i a e}{ }^{61}$, au point de pouvoir émettre une critique à leur sujet. Il en connaissait même peut-être d'autres, qui ne sont point parvenues jusqu'à nous. Ce même souci littéraire transparaît dans le paragraphe sur Charles le Chauve : l'auteur profite de l'occasion pour mentionner un abrégé de la vie de cet empereur, qu'avait fait rédiger sa nièce Bertrade. Visiblement, nous avons affaire à un individu ayant des connaissances littéraires assez étendues, bien plus qu'en aurait un simple copiste. Chacun des deux paragraphes ajoutés fait allusion à des œuvres de styles différents, composées à des endroits relativement éloignés: Reims et Faremoutiers, au sud-est de Meaux. S'il était uniquement un copiste de Reims, il pouvait éventuellement connaître les œuvres de Flodoard, chanoine de cette église, mais beaucoup plus difficilement celle due à Bertrade. Par ailleurs, le style travaillé des obits de Charles le Chauve et de Flodoard dénote d'aptitudes littéraires particulières.

Ce copiste érudit avait donc probablement l'intention d'emmener la copie de cette œuvre dans sa communauté. Il ne donne malheureusement aucune indication sur ce lieu. Cependant, cette copie était à Laon au plus tard en 977 : le continuateur

60. Les dernières années des Annales sont plus sèches, comportent des erreurs et redondances inhabituelles auparavant, si bien que Philippe Lauer observe un affaiblissement des «facultés intellectuelles de l'annaliste», qui s'avoue lui-même vieillissant et touché d'infirmités (FLODOARD, p. XVIII-XIX).

61. Sur les Triomphes du Christ et l'Histoire de l'Église de Reims, voyez Sot, 1993, p. 87-101 et 101-107. 
a donc pu copier les Annales à Reims vers le mois de juillet 966 pour les amener ensuite dans cette ville. Suivant cette hypothèse, voyons s'il est possible d'identifier un éventuel copiste auteur de ces additions.

\section{Hypothèse d'identification de l'auteur des additions portant sur les années 877 et 966}

Face au manque d'informations à notre disposition, attribuer un nom à ce copiste n'est pas tâche aisée. En 966, l'archevêque de Reims était Odelric (962-969), et à Laon, l'évêque était Roricon (949-976). Ce dernier, fils naturel de Charles le Simple, s'opposa au rétablissement d'Hugues de Vermandois sur le siège de Reims après la mort de l'archevêque Artaud en $962^{62}$. Il soutint ouvertement la candidature d'Odelric, qui fut consacré cette même année : des liens étroits existaient donc vraisemblablement entre les deux hommes. Roricon fut également chancelier de son demi-frère le roi Louis IV d'Outre-Mer entre 943 et 949, avant de devenir évêque ${ }^{63}$. Grâce à ce poste, il fut probablement présent aux côtés de Louis IV lors des différents synodes relatifs à l'archevêché de Reims en 947 et 948, notamment celui d'Ingelheim où fut également présent Flodoard ${ }^{64}$. Il put aussi bien être auditeur de l'événement en tant que diacre accompagnant son évêque, puisqu'il occupait aussi cette fonction à cette époque ${ }^{65}$. Ainsi, Roricon connaissait vraisemblablement Flodoard, chanoine réputé et incontournable de l'Église de Reims, au moins dès $948^{66}$.

62. SASSIER, 1987, p. 152. L'argument de défense avancé par Roricon laisse supposer sa présence au concile d'Ingelheim de 948.

63. Recueil des actes de Louis IV roi de France (936-954), p. XV. Recueil des actes de Lothaire et de Louis $V$, rois de France (954-987), p. VII, $\mathrm{n}^{\circ} 43$ et acte $\mathrm{n}^{\circ}$ XIII, p. 29. Roricon (Rorgon) fut notaire et chancelier de son demi-frère le roi Louis IV d'Outre-Mer sous plusieurs archichanceliers différents: l'archevêque de Reims Hugues de Vermandois (actes du 10 août 943 et du 11 février 945), l'archevêque de Bourges Géronce (actes du 4 mars 944, 10 juillet 944 et du 29 septembre 948) et l'archevêque de Reims Artaud (acte du 26 juin 945). Il est également mentionné dans un

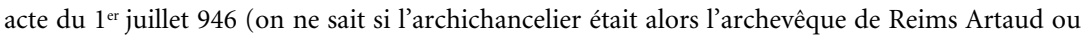
l'évêque de Langres Achard). L'acte du 29 septembre 948 est le dernier où il apparaît sous ce titre, et il était évêque de Laon dès le début de l'année suivante: il est probable qu'il a abandonné sa charge de chancelier en montant sur son siège épiscopal. Toutefois, Roricon apparaît sous le règne de Lothaire à la place de l'archichancelier Artaud dans un acte du 10 décembre 960, sans toutefois porter ce titre: le remplacement exceptionnel de l'archevêque de Reims par l'évêque de Laon s'explique probablement par l'incapacité du premier à pouvoir exercer cette tâche. Artaud devait d'ailleurs mourir moins d'un an après, le 5 septembre 961.

64. Sur ce concile d'Ingelheim, réunissant en 948 de nombreux évêques en présence des rois Otton Ier et Louis IV, voyez FLODOARD, an. 948 (p. 109 et suiv.) et SASSIER, 1987, p. 117.

65. Roricon fut diacre, vraisemblablement de l'église de Laon, avant de devenir évêque de cette même ville en 949, après le décès de Raoul de Laon (FLODOARD, an. 949 p. 121).

66. L'existence des Annales de Flodoard fut probablement révélée lors du concile d'Ingelheim de 948. L'archevêque Artaud s'appuya fortement sur elles pour rédiger son «libelle», discours qu'il prononça pour sa défense lors de ce concile (sur ce texte, voyez SOT, 1993, p. 266-267, 275-276 et 287-289). Il est probable que Roricon a connu cette œuvre de Flodoard à cette occasion, et c'est à la suite de ce concile que Flodoard fut sollicité par un certain «prélat R.» pour rédiger l'Histoire de l'Église de Reims. 
Nous savons par ailleurs que ce prélat disposait d'un vaste savoir et de grandes connaissances littéraires grâce aux témoignages d'Adson de Montier-en-Der et de Richer de Reims ${ }^{67}$. À tel point que Philippe Lauer ${ }^{68}$, et après lui Michel Sot ${ }^{69}$, voient en lui l'un des personnages possibles à qui Flodoard aurait pu dédier son Histoire de l'Église de Reims derrière l'énigmatique phrase rhétorique «à Monseigneur le vénérable, tout à fait aimable par la charité du Christ et illustre prélat R.». Certes, l'archevêque Robert de Trèves lui est généralement préféré, mais cette hypothèse n'est pas dénuée de fondement. En effet, l'Histoire de l'Église de Reims était présente à Laon dès le début des années 960 comme l'a démontré Heinz Löwe en étudiant un Dialogus de statu sanctae ecclesiae composé par un irlandais sous l'autorité de Roricon ${ }^{70}$. L'évêque de Laon faisait ainsi partie du nombre restreint de privilégiés à connaître cette œuvre peu de temps après la fin de sa rédaction vers 952. S’il n'est pas très surprenant de trouver une copie de l'ouvrage à Laon de façon aussi précoce du fait de la proximité des deux villes, on notera toutefois l'intérêt particulier de ce prélat pour cette œuvre de Flodoard. À la vue de cette attention portée sur l'Histoire de l'Église de Reims, son attrait pour les autres œuvres de Flodoard, afin d'en doter la bibliothèque de son Église de Laon, ne semble pas inconcevable. Son désir d'obtenir une copie des Annales peu après la mort de Flodoard paraîtrait dans la logique d'une telle démarche, surtout s'il avait eu connaissance de son existence auparavant. Érudit, carolingien et chancelier du roi, Roricon connaissait vraisemblablement également l'abrégé de la vie de son arrière-grand-père, Charles le Chauve, que fit rédiger sa parente Bertrade, abbesse de l'abbaye royale de Faremoutiers ${ }^{71}$.

Quant aux additions, soulignons l'intérêt que Roricon aurait pu leur accorder. L'année 877 concerne ses ancêtres empereurs, dont son arrière-grand-père Charles le Chauve et les père et grand-père de celui-ci (voir figure 1). Dans l'hypothèse où Roricon en fut l'auteur, il aurait alors fait preuve d'une mémoire familiale sélective coutumière à cette époque ${ }^{72}$. Sa connaissance des œuvres de Flodoard le prédisposait

67. SOT, 1993, p. 102; "Roricon, historien et autres écrivains", in Histoire Littéraire de la France, t. VII, p. 186: «totius scientiae lumen». Richer (II, 82, p. 268-269) écrit: «Rorico, omni rerum scientia inclitus».

68. FlodoARD, p. XIV-XV; Recueil des actes de Louis IV roi de France (936-954), p. X et suiv.

69. Sот, 1993, p. 102.

70. Ibidem, p. 102.

71. Roricon apparaît en 962 (FlODOARD, an. 962, p. 151; SASSIER, 1987, p. 152) dans un synode de treize évêques des diocèses de Reims et de Sens tenu dans le Meldois, sur les bords de la Marne: il n'était donc pas très loin de Meaux et de l'abbaye royale de Faremoutiers. Serait-ce vers cette époque qu'il aurait pu prendre connaissance de l'obituaire de cette abbaye ou/et de l'abrégé de la vie de Charles le Chauve?

72. Régine Le Jan (1995, p. 35, 43-44 et 57) indique qu'à l'époque carolingienne, sous l'influence du christianisme, «la prière pour les morts, la memoria, s'imposa comme le principal devoir des vivants à l'égard de leurs parents défunts »: «la conscience généalogique pouvait atteindre la génération des arrière-arrière-grands-parents, voire au-delà, dépassant ainsi largement la mémoire biologique. Mais elle était sélective, elle résultait d'un choix opéré au sein de la parenté. [...] La mémoire se cristallisait sur la ligne de parenté «la plus noble », et d'abord sur la parenté royale. 
également à rédiger pour l'année 966 un obit à son sujet en ces termes. Enfin, l'intérêt qu'aurait pu porter Roricon aux Annales semble évident lorsque l'on examine les informations qu'elles renferment sur ses proches - son père Charles le Simple, son demi-frère Louis IV et son neveu Lothaire - ou sur lui-même: son accession à Laon en 949, sa présence lors de la consécration de l'évêque Hadulfe de Noyon en 956; sa participation au siège de La Fère en 958 ; son opposition à Hugues de Vermandois et son soutien à Odelric pour l'archevêché de Reims en 962 ; ou encore son intervention auprès des grands de Flandre en faveur de Lothaire en 965.

Enfin, son statut de carolingien, ex-chancelier de son demi-frère le roi Louis IV, devait lui permettre d'ouvrir certaines portes que d'autres n'auraient pu franchir à sa place: l'accès aux archives de l'Église de Reims et à l'abbaye royale de Faremoutiers ne devait pas poser trop de problème pour lui. On le voit, Roricon avait bien toutes les qualités requises pour être le copiste et l'auteur des additions portant sur les années 877 et 966.

Cependant, nous ne pouvons affirmer avec certitude que les Annales ont bien été copiées par Roricon lui-même, et que les additions sont également de sa main: trop peu d'informations portent sur les années 877 et 966 pour pouvoir identifier ce continuateur de façon certaine. D'autant que la proximité des villes de Reims et de Laon, l'importance des relations entre ces deux cités et le prestige de leurs écoles respectives entre 966 et 976 ne permettent pas de véritablement isoler un érudit particulier, même si peu d'entre eux semblent disposer de toutes les qualités requises exposées ici. Il s'agit donc simplement d'une hypothèse séduisante, guidée par quelques indices allant dans ce sens.

Essayons maintenant de reconstituer comment les Annales parvinrent de Reims - où Flodoard les a écrites - jusqu'à Fécamp par l'intermédiaire de la ville et de l'Église de Laon.

\section{Reconstitution hypothétique du cheminement des Annales de Flodoard de Reims à Fécamp}

Flodoard rédige ses Annales à Reims de 919 jusqu'à sa mort le 28 mars $966^{73}$. À cette date, son œuvre, ainsi que ses autres manuscrits originaux - De triumphis Christi et Historia Remensis Ecclesiae - sont vraisemblablement gardés dans les archives de l'Église de Reims, dont Flodoard avait lui-même eu la charge.

[...] Elle remontait dans le passé généalogique pour se fixer sur les ancêtres les plus « utiles », ceux qui légitimaient le pouvoir et la noblesse de leurs descendants ».

73. FlodoARD, p. XV-XX et XXIV; Sot, Michel, p. 86-87. Si les additions faites par le copiste ont bien eu lieu en 966, l'ambiguïté du nécrologe de Reims au sujet de la date de décès de Flodoard n'existait pas alors. En effet, l'auteur des Annales était bien mort à cette époque, mais son neveu homonyme était, lui, probablement toujours en vie. Une seule date concernant « un Flodoard» devait ainsi exister dans le nécrologe en 966 : le continuateur a donc bien reporté la date exacte de la mort de l'auteur des Annales dans sa continuation, c'est-à-dire le 28 mars. Celle du 17 mai correspond probablement à celle de son neveu, décédé plus tard. 
C'est dans ce contexte que les Annales sont probablement copiées autour du 23 juillet 966. Le copiste ne se contente pas de recopier simplement l'ensemble du texte de Flodoard: il apporte deux additions non négligeables. Au début, il insère un obit sur Charles le Chauve dans un paragraphe consacré à l'année 877 et mentionne l'œuvre que fit rédiger à son sujet sa nièce Bertrade. À la fin, il ajoute, après l'année 966, la rubrique Hucusque Cronica Flodoardi Presbiteri pour finaliser la partie écrite par Flodoard et il complète cette année d'un obit sur l'auteur, où il fait allusion à ses autres œuvres. Il mentionne également les importantes inondations survenues probablement durant la période où il copiait ces Annales. Une fois l'œuvre reproduite et les additions effectuées, le manuscrit est vraisemblablement emmené aussitôt après à Laon : ce copiste est donc probablement un clerc de l'église de cette ville.

Bien que nous n'en ayons pas la certitude, il semble que l'auteur de cette copie et de ces additions puisse être l'évêque Roricon de Laon. Il a pu dupliquer lui-même l'œuvre originale, à moins qu'il ait chargé un scribe de l'Église de Reims - ou de Laon - de ce travail fastidieux. Il était surtout bien placé pour rédiger les deux additions portant sur les années 877 et 966, puis pour emmener ce manuscrit de Reims à Laon.

À la mort de l'évêque Roricon, Adalbéron est nommé par Lothaire, pour lui succéder sur le siège épiscopal de Laon. Il avait auparavant été formé par son oncle homonyme dans l'école cathédrale de Reims, qui jouissait alors d'une excellente réputation, grâce notamment aux cours dispensés par l'archidiacre Gerannus ${ }^{74}$, et l'écolâtre Gerbert d'Aurillac ${ }^{75}$. Fort de cette formation et aidé par ses origines, Adalbéron est ensuite appuyé par son oncle, archevêque de Reims et archichancelier du roi Lothaire, pour devenir lui-même chancelier du roi de France vers $974^{76}$. Une fois devenu évêque de Laon en 977, il prend connaissance de la copie des Annales présente dans la bibliothèque de cette église. Ce manuscrit est intéressant pour lui à plusieurs titres. En tant que chancelier du roi Lothaire, il trouve nombre d'informations sur les événements survenus dans le royaume depuis le début de son siècle, et en particulier sur la province de Reims, dont dépend son évêché de Laon. Mieux,

74. Sur Gerannus, voyez LOT, 1891 p. 76-77; ADALBÉRON DE LAON, p. XIV. Gerannus était archidiacre et écolâtre de l'école de Reims vers 970 où il enseignait la logique (philosophie).

75. Sur Gerbert, voyez GERBERT, p. I-XXXVIII ; LOT, 1891, p. 76-77; PARISSE, "Gerbert d'Aurillac », in Dictionnaire du Moyen-Âge, p. 585; "Sylvestre II, pape», in Histoire Littéraire de la France, t. VI, p. 559-614. D'origine modeste, Gerbert connut un destin unique pour son époque et gravit grâce à son savoir et ses connaissances, les différents échelons de l'Église, commençant simple oblat et finissant premier pape français sous le nom de Sylveste II (999-1003), après avoir été respectivement écolâtre de l'école de Reims (972-982) - où il professa un enseignement très réputé tout en étant secrétaire de l'archevêque Adalbéron -, abbé laïc de Bobbio (v. 982/983), archevêque de Reims (991-998) puis de Ravenne (998-999).

76. Recueil des actes de Lothaire et de Louis V, rois de France (954-987), p. VII-IX et actes ${ }^{\circ}$ XXXIVXLII p. 83-100 ; ADALBÉRON DE LAON, p. XI ; COOLIDGE, 1965, p. II ; SASSIER, 1987, p. 161. Les actes datés, où Adalbéron de Laon apparaît comme notaire et chancelier royal de Lothaire, sont de 974 et 975 ; les autres actes non datés ont probablement été rédigés entre 972 et 979, sans que l’on puisse préciser davantage. 
il l'intéresse surtout personnellement : tous les faits marquants touchant à sa région d'origine, en Lotharingie, sont relatés sur la période couverte par les Annales. Enfin, il est question de ses ancêtres carolingiens - dans l'addition faite par le premier continuateur en 877 - et de plusieurs membres de sa famille d'Ardenne, dont les évêques Wigeric et Adalbéron de Metz ${ }^{77}$, le duc Frédéric (Ferry) de Haute-Lorraine ${ }^{78}$, ou encore l'archevêque de Reims Odelric ${ }^{79}$ (voir figure 1).

C'est probablement lui qui entreprend de poursuivre ces Annales à cette époque (à moins qu'il s'agisse de l'un de ses proches disciples). Il note une succession d'années sans mention de 967 à 975, puis commence par plusieurs événements qui se sont déroulés l'année précédente, en 976 . Toutes ses notes concernent soit directement ses proches parents de la "Maison d'Ardenne», soit son évêché de Laon: la première désapprouve vigoureusement les travaux entrepris dans l'Église Sainte-Marie par son oncle l'archevêque de Reims; la seconde relate la bataille survenue en Lorraine pendant le carême, impliquant son oncle Godefroi le Captif et son cousin par alliance Charles (voir figure 1). Puis, arrivant à l'année en cours, il relate les différentes étapes de son accession à l'évêché de Laon dans tous leurs détails, après avoir rappelé le décès de l'évêque Roricon à la fin de l'année précédente. Il indique également une information sur un évêché voisin de celui de Laon en relatant le décès de l'évêque Hadulfe de Noyon ${ }^{80}$. En mentionnant la mort de Mac Allan survenue en 978, il rap-

77. Sur Wigeric: FlodoARD, an. 923 (p. 17 et 18) et an. 927 (p. 37). Sur Adalbéron: FlodoARD, an. 929 (p. 44), an. 943 (p. 89), an. 948 (p. 107, 110, 115), an. 950 (p. 127) et an. 951 (p. 130). Wigeric, évêque de Metz de 919 à 927, était probablement un parent de la «Maison d'Ardenne », comme le suggère son nom peu répandu (voir PARISSE, 1976, p. 21). Le duc Frédéric Ier indique la générosité de ce parent probable dans un acte de 959 (PARISSE, 1993, p. 55). Initialement vivement opposé d'Otton I ${ }^{\text {er }}$, Adalbéron de Metz (929-962) fut parmi les derniers évêques de Lotharingie à accepter ce roi de Germanie (PARISOT, 1919, p. 187). Mais lorsque cela fut fait, son attachement pour Otton se révéla sans faille. Il intervint dans les affaires de l’Église de Reims en 948 lors de synodes débattant de la légitimité des archevêques Hugues de Vermandois et Artaud, puis dans ceux opposant le roi de France Louis IV d'Outremer au duc de France Hugues en 950-951.

78. FlODOARD, an. 951 (p. 131), an. 954 (p. 139), an. 959 (p. 147), an. 960 (p. 148) et an. 965 (p. 157, $\mathrm{n}^{\circ} 2$ ). Frédéric (Ferry) épousa Béatrice (Béatrix), une fille d'Hugues le Grand en 954 et devint duc de Haute-Lorraine en 959.

79. FlodOARD, an. 962 (p. 151), an. 964 (p. 155), an. 965 (p. 156) et an. 966 (p. 158). Odelric fut élevé à Metz par Adalbéron, évêque de cette ville, où il devint chanoine avant de devenir abbé laïc en 942. Il succède à Artaud à la tête de l'archevêché de Reims en 962. Ferdinand Lot (1891, p. 50, note 1) indique que la mère d'Odelric, Eva, était consanguinae avec l'évêque Adalbéron de Metz, ce qui signifie probablement « cousine ». Il nous est malheureusement impossible de déterminer à quel niveau ce lien de parenté était établi. Il est fort probable qu'Odelric soit lié aux carolingiens par son père Hugues - descendant de Saint-Arnoul, évêque de Metz - et à la "Maison d'Ardenne » par sa mère Eva (nièce de Wigeric?). Sur le terme consanguineus, voyez LE JAN, 1995, p. 166, 173-174 et 176. On notera que les informations des Annales sur Ricuin (an. 921, p. 6, an. 923, p. 13), second époux de Cunégonde, et sur son fils Otton (an. 922, p. 7, an. 923, p. 18, an. 942, p. 85, an. 943, p. 89, an. 944, p. 91), proches de la «Maison d'Ardenne», pouvaient elles aussi intéresser Adalbéron.

80. Hadulfe, évêque de Noyon, avait soutenu et consacré Odelric archevêque de Reims en 962 : celui-ci fut le premier archevêque lié à la famille d'Ardenne. Cette famille accédait ainsi à un nouveau rang ecclésiastique et accroissait son prestige au-delà de la Meuse, hors de la Lotharingie, où elle n'avait pas encore d'évêché. 
porte une information intéressante pour son Église de Laon, puisque les monastères de cet abbé dépendent de cet évêché.

Soudain, la continuation des Annales s'interrompt. Pourquoi avoir stoppé aussi brusquement cette rédaction? En 977, peu après la nomination d'Adalbéron à l'évêché de Laon, un scandale secoua la cour du roi: le propre frère de Lothaire, Charles, accusa ouvertement sa belle-sœur, la reine Emma, d'adultère avec l'évêque Adalbéron de Laon, son cousin par alliance ${ }^{81}$. Devant le manque de preuves du dénonciateur, le concile de Saint-Macre, dirigé par l'archevêque de Reims, disculpa les accusés et Lothaire bannit son frère du royaume : ce dernier se réfugia en Lorraine ${ }^{82}$. C'est dans ce contexte qu'Otton II nomma Charles duc de Basse-Lorraine et en fit son vassal. Lothaire, furieux ${ }^{83}$, fit une expédition contre l'empereur, dans le but de s'en emparer à Aix-la-Chapelle. Ainsi, l'ost royal lança un raid éclair contre le palais impérial au début du mois d'août 978, et faillit réussir dans cette audacieuse entreprise: Otton II, surpris, fut contraint de fuir précipitamment vers Cologne. N'étant pas parvenu à ses fins, Lothaire rentra en France, où de terribles représailles ne tardèrent pas à suivre: Otton II rassembla une armée considérable dès septembre 978 et commença à envahir les terres du roi de France début octobre. Il ravagea le Laonnois, le Soissonnais et le Rémois, tandis que les palais royaux de Compiègne et d'Attigny étaient pillés et détruits. Lothaire fut contraint de fuir lorsque la ville de Laon fut prise et Charles proclamé roi dans cette cité par l'évêque Thierry de Metz, sur ordre d'Otton II ${ }^{84}$. Ce dernier marcha ensuite jusqu'à Paris où Lothaire s'était réfugié auprès $\mathrm{du}$ duc de France, Hugues Capet. Le roi carolingien, à cet endroit, reçut alors le soutien d'une puissante armée venue d'Anjou, de Bourgogne et de Francie : arrêtés devant Paris, l'empereur et son armée furent contraints de faire demi-tour. Poursuivis par l'armée des Francs, ils rentrèrent précipitamment jusqu'en Lorraine, emmenant avec eux Charles, obligé de quitter Laon ${ }^{85}$. Les différends entre le roi de France et

81. À cette époque, Charles avait épousé Bonne d'Ardenne, fille du comte Godefroi (voir figure 1 et 2). Celle-ci mourut avant 979. C'est probablement aussi du fait de ce mariage qu'Otton II donna le duché de Basse-Lorraine à Charles. On notera que le premier enfant issu de cette union fut prénommé Otton. Au sujet des choix de nom à cette époque, voyez LE JAN, 1995, p. 179-223, et en particulier p. 221-222.

82. SASSIER, 1987, p. 162 ; GERBERT, p. XX. Voyez aussi la version du synode de Saint-Macre faite par RICHER (III, 66, p. 80-81).

83. Reprenons ici les termes d'Yves Sassier (1987, p. 162) commentant le don du duché de Basse-Lorraine à Charles par Otton II : «Honorer ainsi celui qui a voulu jeter opprobre et déshonneur sur l'épouse du roi des Francs, c'est faire offense au roi des Francs lui-même».

84. L'évêque Thierry de Metz (965-984) était cousin germain de l'archevêque Brunon de Cologne et de l'empereur Otton Ir (PARISSE, p. 84). Il était le fils du comte Eberhard et d'Amalrade, sœur de la femme d'Henri Ier l'Oiseleur, Mathilde (GERBERT, p. 25 n. 2 ; LE JAN, 1995, p. 44-45, 83-84). Bien que couronnant Charles à Laon en 978, il aura à l'égard de celui-ci des propos très durs dans une lettre rédigée six ans plus tard (SASSIER, 1987, p. 197), notamment sur son comportement à l'égard de la reine Emma et d'Adalbéron de Laon.

85. Sur ces événements survenus en 977-978, voyez SASSIER, 1987, p. 161-165; LE JAN, Régine, Histoire de la France, p. 152. Voyez également LOT, 1891, p. 91-103 et 106-108; POULL, 1994, p. 7-16. Voir aussi la version de RICHER (III, 68-77, p. 82-97). 
l'empereur se prolongèrent pendant près de deux ans, avant qu'une paix fragile ne soit conclue en $980^{86}$.

On remarquera tout d'abord que les événements compromettants concernant l'évêque de Laon ne sont pas mentionnés dans la continuation des Annales en 977 : un tel silence ne peut s'expliquer que chez un scribe partisan de l'évêque. De plus, on comprend aisément qu'Adalbéron ait dû fuir précipitamment de sa ville en 978 sans emporter les Annales avec lui : il était chancelier de Lothaire et il accompagnait très certainement le roi dans son repli sur Paris. Il n'avait probablement pas encore embrassé les partis robertien ou ottonien comme il le fit plus tard ${ }^{87}$. Cette période marque cependant un tournant décisif dans les relations entre les Carolingiens, les Robertiens et les Ottoniens. Et la «Maison d'Ardenne» se trouva dès lors très impliquée dans les enjeux politiques la liant à ces trois familles ${ }^{88}$ (voir figure $2^{89}$ ). Si des tensions existaient déjà depuis longtemps au sujet de la Lotharingie, l'action de Lothaire contre l'empereur Otton II réduisit à néant le fragile équilibre qui subsistait encore entre les deux pays depuis la mort d'Otton $I^{\text {er }}(973)$ : par cet acte, le roi de France venait d'affirmer clairement sa décision de s'affranchir de la tutelle de l'Empire. C'est, semble-t-il, vers cette époque que les derniers membres de la «Maison d'Ardenne » se sont irrémédiablement détournés de Lothaire pour aller vers les Robertiens et les Ottoniens de Germanie, s'ils ne l'avaient déjà fait ${ }^{90}$. Nous ne discute-

86. Lothaire se rapprocha d'Otton II en juillet 980, par le traité de Margut-sur-Chiers (SASSIER, 1987, p. 167). Après la mort de celui-ci en 983, les hostilités reprendront, d'abord pour la tutelle du jeune Otton III en 984 (Ibidem, p. 173-175), puis par la prise de Verdun en 985 au cours de laquelle plusieurs membres de la « Maison d'Ardenne » furent faits prisonniers par Lothaire (Ibidem, Yves, p. 179-180).

87. À ce sujet, voyez Ibidem, p. 231-235 et 251-255; RICHER, IV, 97-98, p. 307-313 et IV, 41-49, p. 204-225; GERBERT, p. XXIV.

88. LE JAN, 1995, p. 291, 294-295, 302, 324. La famille d'Ardenne descendait des carolingiens par Cunégonde, fille d’Ermentrude et petite-fille de Louis le Bègue. En 954, Frédéric, comte de Verdun, épouse Béatrice, fille de Hugues le Grand, duc de France et abbé laïc de Saint-Denis. Ce mariage le fait entrer dans la famille des Robertiens - il devient beau-frère d'Hugues Capet et oncle de Robert le Pieux, futurs rois de France - mais également dans celle des Ottoniens : la mère de Béatrice est Hadwige (Hathui/Judith) de Saxe, fille d'Henri I ${ }^{\text {er }}$ l'Oiseleur, et sœur d'Otton I. Cette situation lui vaudra d'obtenir le duché de Haute-Lorraine en 959, de la part de Brunon de Cologne, frère d'Ot-

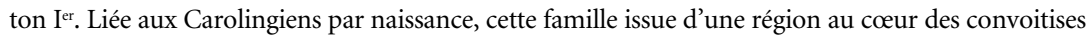
(la Lotharingie), se retrouve par mariage plus proche des Robertiens et des Ottoniens que des rois carolingiens: en 959, Hugues Capet est le beau-frère de Frédéric, l'Empereur Otton ${ }^{\mathrm{er}}$ est son oncle par alliance, alors que Lothaire n'est qu'un cousin très éloigné! D’autre part, le frère de Frédéric, Gozlin, épousa Uda de Metz, dont la mère Uda de Saxe était une sœur d'Henri I ${ }^{\text {er }}$ l'Oiseleur. Leurs enfants, Adalbéron de Reims et Godefroi le Captif, se retrouvaient ainsi rattachés aux Carolingiens par leur grand-mère paternelle Cunégonde, et aux Ottoniens par leur grand-mère maternelle, Uda de Saxe : ils étaient ainsi petits-cousins d'Otton $\mathrm{I}^{\mathrm{er}}$. En se mariant avec Bonne, fille du comte Godefroi le Captif, Charles deviendra lui-même plus proche de la famille d'Ardenne que son propre frère le roi Lothaire. On comprend les enjeux politiques complexes tournant autour de cette famille lors des oppositions entre ces trois dynasties (voir figure 1 et 2).

89. LE JAN, 1996, p. 240; LE JAN, 1995, p. 221 et 414-416.

90. Yves Sassier (p. 167) souligne que «le métropolitain de Reims et son neveu de Laon [...] n’ont guère apprécié l'incartade lorraine de 978 » menée par Lothaire. De même, un peu plus loin (p. 180) : «Un 


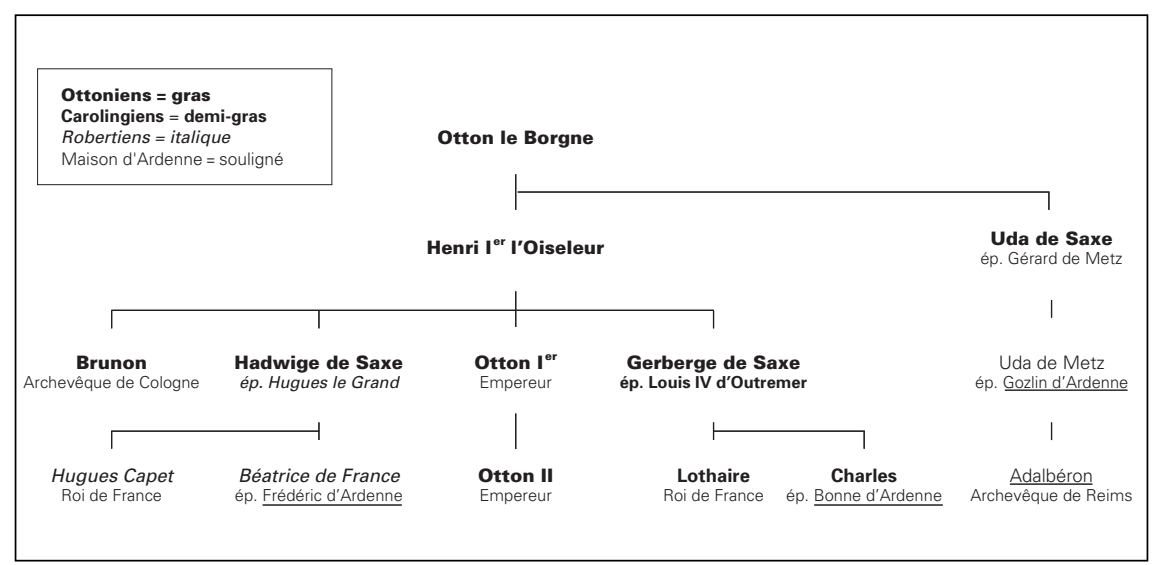

Figure 2: Liens des Ottoniens avec les Carolingiens, les Robertiens et la Maison d'Ardenne.

rons pas ici des détails complexes de cette période particulièrement troublée ${ }^{91}$. Nous indiquerons simplement qu'Adalbéron de Laon lui-même joua un rôle essentiel dans la chute des derniers Carolingiens aux côtés de son oncle homonyme archevêque de Reims et de Gerbert d'Aurillac ${ }^{92}$. Ces conflits et cette activité politique intense permettent toutefois d'expliquer certains silences dans la continuation des Annales, ainsi que leur interruption. Le scandale de l'adultère entre la reine Emma et l'évêque Adalbéron resurgit à plusieurs reprises par la suite ${ }^{93}$ et la ville de Laon, qui s'était imposée comme capitale-forteresse des rois carolingiens Louis IV d'Outre-Mer et Lothaire ${ }^{94}$, fut exposée aux convoitises de Charles de Basse-Lorraine

Adalbéron ne peut concevoir la survie de la royauté carolingienne qu'à condition que celle-ci accepte le second rôle qui lui revient dans le cadre d'un empire chrétien dont le gouvernement appartient désormais à la dynastie saxonne».

91. Pour en savoir plus à ce sujet, voyez SASSIER, 1987, p. 165 et suiv. Voyez également LOT, 1891, p. 80-282 ; LOT, 1903, p. 28-90; GERBERT, p. I-XXXVIII ; ADALBÉRON DE LAON, p. IX-XI. Pour une étude sociologique de l'époque, voyez Le Jan, 1995.

92. La correspondance de Gerbert d'Aurillac montre le rôle politique joué par ces trois hommes dans l'avènement de Hugues Capet et la fin du $\mathrm{X}^{\mathrm{e}}$ siècle. En sacrant Hugues, l'archevêque Adalbéron de Reims apporta la caution religieuse au nouveau roi et à la nouvelle dynastie naissante. En livrant à Hugues Capet le dernier carolingien prétendant au trône, son neveu l'évêque Adalbéron de Laon mit irrémédiablement fin aux prétentions de cette dynastie à la couronne de France. Voyez SASSIER, 1987, p. 181. Reprenons ici les termes de Michel Parisse (1976, p. VI) : «De 978 à 987, les dernières années des rois carolingiens de France, la fin d'Otton II et le début du règne d'Otton III permettent de ressentir mieux qu'à toute autre période la position originale de la Lorraine. Ce pays est alors un enjeu entre le Royaume et l'Empire. Le roi français Lothaire entend reprendre la Francia media à son cousin germain, qui est aussi son beau-frère. C'est à Metz et à Verdun que s'organise la défense au profit de l'Empire; c'est avec les évêques, une duchesse d'origine française [Béatrice, fille d'Hugues le Grand] qui la conduit. À côté d'eux, il faut aussi compter Adalbéron, l'archevêque de Reims et le savant Gerbert. »

93. LOT, 1891, p. 70 et suiv.; SASSIER, 1987, p. 188-189; GERBERT, lettre nº 97 p. 89 ; RICHER, IV, 1617, p. 168-175

94. SAINT-DENIS, «Laon», in Dictionnaire du Moyen-Âge, p. 816. 
dans les années qui suivirent ${ }^{95}$. Politiquement très impliqué, il devenait sans doute risqué d'exprimer ouvertement ses idées et ses actions dans la continuation des Annales!

Il semble toutefois qu'Adalbéron ait réussi à maintenir l'enseignement dans son école cathédrale très réputée durant la majeure partie de cette période troublée ${ }^{96}$. Serait-ce là que Dudon aurait reçu sa formation? Les avis sur la question divergent. Si l'origine de Dudon, issu de la petite noblesse du Vermandois, et le contenu de sa formation fortement marquée par la culture carolingienne ne sont que rarement mises en cause, en revanche, le lieu où il reçut cette formation continue à poser problème. La préface dédicatoire de l'œuvre de Dudon semble sous-entendre qu'Adalbéron de Laon fut son maître et nombre d'historiens se sont ralliés à cette hypothèse ${ }^{97}$. Certains préferent simplement indiquer une influence intellectuelle des écoles de Laon et de Reims ${ }^{98}$, tandis que d'autres vont jusqu'à émettre l'hypothèse d'une formation dans la métropole par Gerbert d'Aurillac ${ }^{99}$. Leah Shopkow a mis en avant plusieurs raisons mettant fortement en doute cette dernière hypothèse et préfere envisager une formation de Dudon dans un centre carolingien traditionnel tel que Liège ${ }^{100}$. Nous ne discuterons pas ici plus en détail du lieu de formation de Dudon, ce qui serait hors de propos, mais nous ferons simplement remarquer que, par ce manuscrit, Dudon se retrouve une fois encore rattaché à la ville et à l'Église de Laon.

Serait-ce au cours d'une éducation et d'une formation reçue à Laon, à une date difficile à déterminer, située entre 972 et $987^{101}$, que Dudon aurait pris connaissance

95. SASSIER, 1987, p. 212-221 ; RICHER, IV, 18-23, p. 174-181 ; GERBERT, 121 p. 110, 124-125 p. 113 , 131 p. 118,135 p. 122 et 137 p. 123.

96. Sur cette école de Laon, voyez SoT, 1993, p. 58-60.

97. Henri Prentout (1916, p. 17-19) déduit implicitement de la préface dédicatoire du De moribus qu'Adalbéron de Laon fut sans doute le protecteur et maître de Dudon, sans véritable démonstration; nombre d'historiens l'ont suivi depuis. Mathieu Arnoux (RAOUL GLABER, p. 16) souligne que Dudon n'a gardé de son maître, l'évêque Adalbéron de Laon, qu'une langue d'une infinie préciosité.

98. Pierre Bouet («Dudon de Saint-Quentin», in Dictionnaire du Moyen-Âge, p. 452-453) indique qu'il fut formé en pays Vermandois sous l'influence intellectuelle de ces deux écoles.

99. Partant du principe que Dudon ne put recevoir l'étendue de ses connaissances uniquement à Saint-Quentin, B. Vopelius-Holtzmann a supposé que Dudon aurait étudié à Reims lorsque Gerbert d'Aurillac fut écolâtre de l'école cathédrale (voyez SHOPKOW, 1989, p. 21, col. 2).

100. Ibidem, p. 22-27. Pour plus d'informations sur Liège à cette époque, voyez Histoire de la principauté de Liège (p.17-23 ; 63-64) et surtout KUPPER, 1981.

101. Dudon devait avoir entre vingt et vingt-cinq ans lorsqu'il fut envoyé en Normandie par Albert de Vermandois en 987 (SHOPKOW, 1989, p. $36 \mathrm{n}^{\circ} 24$ ) : il avait donc entre dix et quinze ans en 977. Dans l'hypothèse d'une éducation et d'une formation à l'école cathédrale de Laon, celles-ci lui auraient donc été inculquées sous Adalbéron de Laon (977-1031) peu de temps après son accession, et peut-être même initialement sous Roricon (949-976). À l'époque de ce dernier, Laon était encore un centre carolingien traditionnel proche de la cour royale. L'enseignement fut ensuite peu à peu réformé sous l'influence d'Adalbéron, qui avait été formé par Gerbert d'Aurillac à Reims suivant de nouvelles méthodes d'étude (Ibidem, p. 21, col. 2 p. 22, col. 2). Or on retrouve chez Dudon cette double influence: la première, classique, au travers des œuvres utilisées mises en avant par Leah Shopkow; la seconde, où l'usage de la rhétorique était de rigueur, apparaît en permanence tout au long du récit, par l'emploi d'un langage très travaillé, souvent pompeux, où la recherche d'effets de style et les hyperboles sont omniprésentes. 
de l'existence des Annales de Flodoard? Est-ce simplement grâce à des liens d'amitié entretenus avec l'évêque Adalbéron de Laon qu'il put acquérir cette œuvre? Dans l'état actuel de nos connaissances, nous ne pouvons nous prononcer. Ce qui est sûr, en revanche, c'est que Dudon a entretenu une relation privilégiée avec la ville de Laon, son église et son évêque.

Après sa formation, Dudon devient chanoine de la collégiale de Saint-Quentin, sous l'autorité de l'abbé laïque Albert, comte de Vermandois ${ }^{102}$. Au moment du couronnement d'Hugues Capet, probablement durant l'été 987, Dudon est envoyé en ambassade auprès du duc Richard Ir de Normandie (943-996) par ce même comte, quelques mois avant sa mort ${ }^{103}$. Cette mission lui permet d'entretenir dès lors des rapports privilégiés avec les ducs normands, leur famille et leur cour située à Fécamp. Vers 994, Richard Ire émet la volonté de disposer d'une histoire des ducs de Normandie et sollicite Dudon pour cette raison ${ }^{104}$. Cette œuvre politique doit entre autres légitimer le lignage des ducs ${ }^{105}$ et la possession de la Normandie, en montrant que l'engagement initial, notamment religieux, conclu entre l'ancêtre Rollon et le roi carolingien Charles le Simple a toujours été respecté. C'est dans ce contexte que Dudon se rend à Laon, proche de Saint-Quentin, afin de copier les Annales de Flodoard et qu'il ramène cette copie en Normandie sous le titre de Gesta Normannorum $^{106}$. Et c'est à partir de Fécamp que cette copie des Annales apportée par Dudon fait souche par la suite: au moins quatre copies en sont issues plus ou moins directement et constituent ce que l'on appelle «la branche normande» des Annales de Flodoard.

\section{Conclusion}

Le manuscrit primitif de «la branche normande» des Annales de Flodoard fut vraisemblablement copié à Laon et amené à Fécamp par le chanoine Dudon de Saint-

102. LOT, 1891, p. 215-216; DUDON DE SAINT-QUENTIN, p. 295; SASSIER, 1987, p. 208. Version électronique de Dudon par Felice Lifshitz, chap. 59.

103. Dudon DE SAINT-QUentin, p. 119 et suiv. Version électronique de Dudon par Felice Lifshitz, préface dédicatoire, chap. 1.

104. DudOn DE SAINT-QUentin, p. 119 et suiv. Version électronique de Dudon par Felice Lifshitz, préface dédicatoire, chap. 1.

105. BOUET, 2002, p. 63-65; SASSIER, 1987, p. 208-209. Régine Le Jan (1995, p. 41) souligne le fait que Dudon a rédigé son œuvre dans le contexte de crise qui suivit la mort de Richard Ir , et que son histoire - l'une des premières histoires généalogiques princières - avait pour but de légitimer et conforter le pouvoir de Richard II.

106. Le manuscrit $\alpha$ est-il différent de celui apporté en Normandie par Dudon ( $\mathrm{ms} \beta$ ) ? Il peut s'agir en effet du même manuscrit si l'on considère que Dudon a pu amener en Normandie le manuscrit de Laon sans en faire une copie préalable. Il aurait alors simplement ajouté le titre Gesta Normannorum sur celui-ci. En effet, il s'agit de la seule différence certaine existant entre ces deux manuscrits dans l'état actuel de nos connaissances: nous sommes dans l'incapacité de pouvoir affirmer qu'il s'agit de deux manuscrits distincts ou d'un manuscrit unique. Pour trancher, un réexamen du manuscrit $B$, issu d' $\alpha$ s'avérerait nécessaire. 
Quentin vers la fin du $\mathrm{X}^{\mathrm{e}}$ siècle, probablement aux alentours de l'année 994 . Une reconstitution hypothétique du cheminement de ces Annales depuis Reims jusqu'en Normandie a pu être établie : elle a permis d'envisager la possibilité de voir en Roricon le copiste du manuscrit apporté à Laon, et surtout l'auteur des additions sur les années 877 et 966 ; elle a également défini Adalbéron de Laon comme le continuateur vraisemblable des Annales pour les années 976 à 978 (voir figure $3^{107}$ ).

Philippe Lauer achève l'introduction de son édition des Annales de Flodoard en désignant la réforme clunisienne comme responsable de la diffusion de celles-ci ${ }^{108}$. En fait, cette étude montre au contraire qu'une bonne partie des manuscrits des Annales n'a pas été copiée par des moines de Cluny. Il est même probable que les Annales de Flodoard étaient encore totalement inconnues de cet ordre dans la première moitié du XI ${ }^{\mathrm{e}}$ siècle : Raoul Glaber, présent à Cluny vers 1031 après avoir séjourné dans de nombreux monastères réformés de Bourgogne, ne les connaissait visiblement pas lorsqu'il rédigea ses Histoires ${ }^{109}$. Six des sept manuscrits ayant atteint notre époque comportent les additions des continuateurs de Flodoard: sans eux, nous ne disposerions que d'un seul manuscrit (ms A). En fait, l'un des plus virulents opposants aux moines de Cluny fut même probablement l'auteur d'une partie des continuations des Annales. En effet, Adalbéron de Laon désigna clairement ces derniers comme principaux perturbateurs de l'ordre du royaume dans son fameux Poème au roi Robert ${ }^{110}$.

Les Annales de Flodoard sont reconnues comme une des sources primordiales pour la connaissance de l'histoire de France du $\mathrm{X}^{\mathrm{e}}$ siècle ${ }^{111}$. Ironie du sort, c'est probablement par l'intermédiaire de deux des personnages les plus critiqués des historiens positivistes ${ }^{112}$ qu'elles nous sont parvenues : d'une part, Adalbéron de Laon, à qui l'on doit vraisemblablement une partie des continuations présentes sur six de

107. Ce graphe s'inspire de celui réalisé par Philippe Lauer à la suite de son analyse (FLODOARD, p. XLV-XLVII).

108. FlodoARD, p. LVII ; LAUER, 1898, p. 522-523.

109. RaOUl Glaber, trad. Arnoux, p. 8-11 et 13-14. Raoul Glaber acheva son œuvre vers 1047 après être passé dans plusieurs abbayes dont Saint-Léger de Champaux, Saint-Germain d'Auxerre (v. 1002?, puis entre 1036 et 1047), La Réôme (entre 1003 et 1010 ?), Saint-Bénigne de Dijon (entre 1016 et 1030 ?), Cluny (v. 1031-1033), Saint-Pierre de Bèze (v. 1035-1036) : malgré tous ces déplacements dans des monastères réformés pour la plupart selon l'ordre de Cluny, aucun fait présent dans son récit ne semble tiré directement des Annales de Flodoard.

110. Dans son Carmen ad Rotbertum regem, Adalbéron met nommément en cause le « roi» Odilon, qui n'est autre que l'abbé de Cluny, et présente une tripartition de l'église et de la société nettement différente de celle exposée par les clunisiens ou le défenseur du monachisme qu'est l'abbé Abbon de Fleury (Apologeticus). Sur ce sujet, voir: ADALBÉRON DE LAON, p. CXIX-CXXXV; MORELle, Laurent, "Abbon de Fleury", in Dictionnaire du Moyen-Âge, p. 1; BARTHÉLEMY, Dominique, "Trois ordres (Théorie des) », in Dictionnaire du Moyen-Âge, p. 1411-1412; SASSIER, 1987, p. 203, 251 et 268-276. 111. SASSIER, 1987, p. 12 et 157; FLODOARD, p. XVIII.

112. Sur cette vision de l'école positiviste: "Adalbéron, évêque de Laon », Histoire Littéraire de la France, 1865, T. 7, p 290-297; LOT, 1891, p. 237-242, 272-277 et 281-282; LOT, 1903, p. 28-30 et 89-90; PREnTOUT, 1916, p. 2-4 et 17-20. Dudon n'est pas mentionné dans leur Recueil des Historiens de la France. 


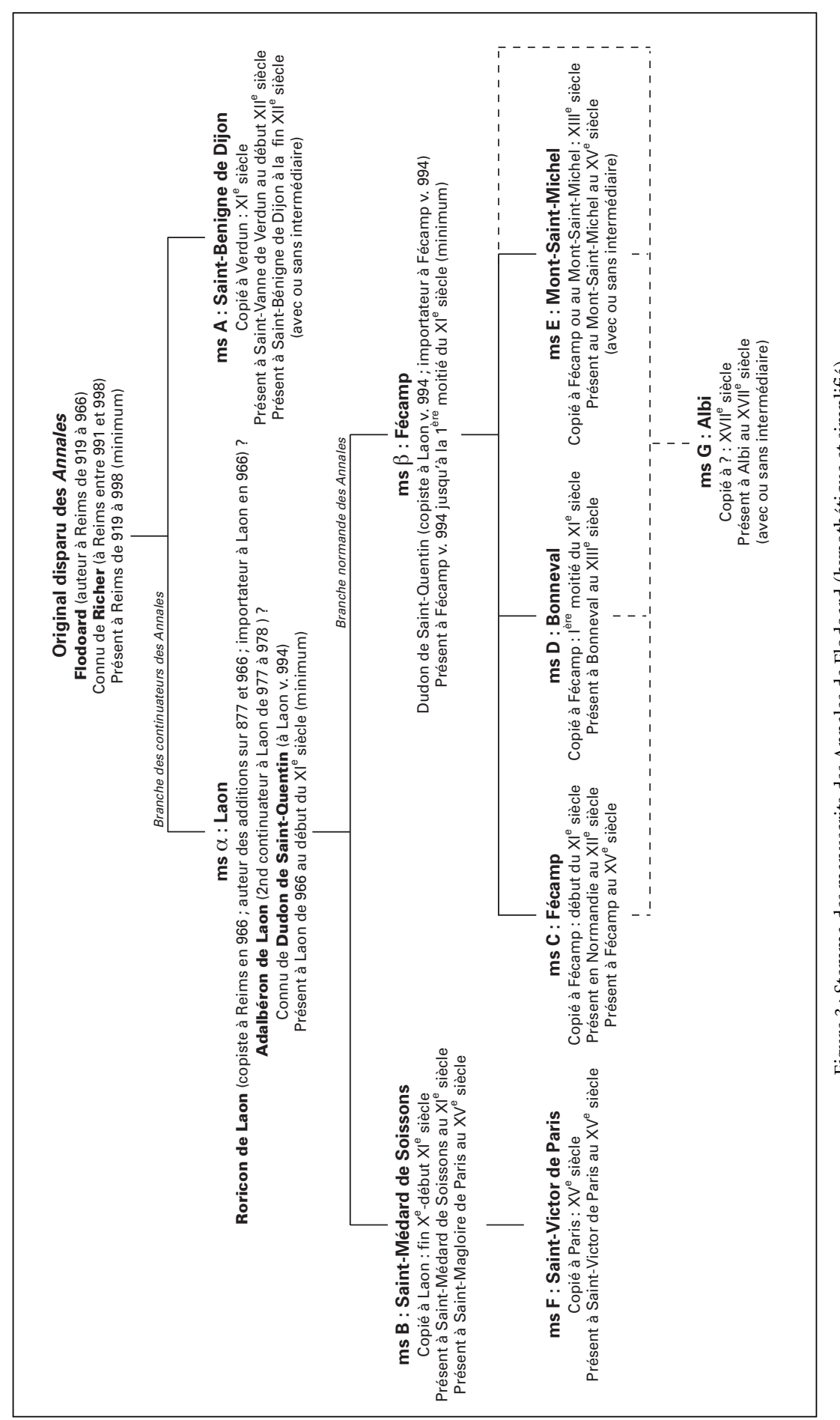

Tabularia «Études », n 4, 2004, p. 1-38, 15 janvier 2004 
nos manuscrits, porte une réputation sulfureuse de traître pour avoir, entre autres, capturé lâchement le dernier Carolingien susceptible de monter sur le trône et pour avoir pactisé avec l'empire ottonien. D'autre part, Dudon, à qui l'on doit indirectement au moins quatre des sept manuscrits (C, D, E et G), fut tellement critiqué pour son style littéraire et ses «fables» qu'il fut ignoré et méprisé par de nombreux historiens ${ }^{113}$.

La mise en évidence de ce nouveau lien unissant Dudon à Laon, et probablement à Adalbéron, nous invite sérieusement à réétudier l'influence de cet évêque sur le doyen de Saint-Quentin. Le fait que la bibliothèque de l'Église de Laon ait fourni à Dudon les Annales, alors que l'évêque de cette même ville est désigné comme le correcteur de son histoire - à qui elle fut dédiée - renforce considérablement l'idée d'une relation maître-élève entre les deux hommes. Face aux différentes connexions reliant le chanoine de Saint-Quentin à la ville et à l'Église de Laon ${ }^{114}$, une analyse minutieuse visant à approfondir la possibilité de son éducation et de sa formation à cet endroit semble plus que jamais s'imposer.

\section{Bibliographie}

Adalbéron de LaOn, Poème au roi Robert, éd. et trad. Claude Carozzi, Paris, Société des Belles-Lettres (Les classiques de l'histoire de France au MoyenÂge, 32), 1979, CLVIII-50 p.

Arnoux, Mathieu, «Before the Gesta Normannorum and beyond Dudo: Some Evidence on Early Norman Historiography», Anglo-Norman Studies, 22, Woodbridge, Boydell Press, 1999, p. 29-48.

BROCKHAUS, Katrin, «La genèse architecturale de l'église de la Trinité de Fécamp », Tabularia «Études», n 2, 2002, p. 71-82.

BouET, Pierre, «Dudon de Saint-Quentin et Fécamp », Tabularia «Études », n² 2, 2002, p. 57-70.

CoOlidge, Robert T., "Adalberon, Bishop of Laon», Studies in Medieval and Renaissance history, 2, Lincoln, University of Nebraska Press, 1965.

Dictionnaire du Moyen-Âge, Claude GaUVARD, Alain DE LIBERA et Michel ZINK (dir.), Paris, Presses Universitaires de France, 2002, LI-1548 p.

DUCHESNE, André, Historiae Normannorum scriptores antiqui, Paris, 1619, p. 49-160.

113. De l'œuvre de Dudon, nous ne disposons que des deux éditions d'André Duchesne et de Jules Lair, ainsi que d'une étude critique faite par Henri Prentout : de nombreux points seraient à revoir dans ces éditions (qui ne portent que sur un nombre réduit de manuscrits, alors que Gerda Huisman en a comptabilisé quinze) et dans cette étude (la vision d'Henri Prentout est très partiale) aujourd'hui dépassées du fait de recherches et découvertes apportées dans divers domaines, notamment en archéologie. Pierre Bouet travaille actuellement sur une nouvelle édition critique qui devrait permettre de faire le point sur l'état de nos connaissances, dont l'intérêt pour l'histoire de la Normandie est primordiale.

114. Pour plus d'information sur la ville de Laon et son église, voyez SAINT-DENIS, 1987, p. 63-135. 
DUdOn De SAINT-QUENTIN, De moribus et actis primorum Normanniae ducum, éd. Jules LAIR, Caen, le Blanc-Hardel (Mémoires de la Société des Antiquaires de Normandie, vol. 23, part. 2), 1865 , p. 115-314.

FlodoARd, Annales, éd. Philippe LAUer, Paris, Picard (Collection de textes pour servir à l'étude et à l'enseignement de l'histoire), 1906, LXX-307 p.

GAZEAU, Véronique, «Guillaume de Volpiano en Normandie: état des questions», Tabularia «Études», $\mathrm{n}^{\circ}$ 2, 2002, p. 35-46.

GERBERT, Lettres, éd. Julien HAVET, Paris, Picard (Collection de textes pour servir à l'étude et à l'enseignement de l'histoire), 1889, LXXXVIII-255 p.

Histoire de la principauté de Liège: De l'an mille à la Révolution, Bruno DEMOULIN et Jean-Louis KuPPER (éd.), Toulouse, Privat (Histoire des provinces), 2002, $271 \mathrm{p}$.

Histoire du Luxembourg: Le destin européen d'un "petit pays », Gilbert TRAUSCH (dir.), Toulouse, Privat (Histoire des provinces), 2002, 333 p.

Histoire Littéraire de la France, éd. Alexis PAULin, Paris, 1865 ( $2^{e}$ édition), 15 vol.

Huisman, Gerda C., "Notes on the manuscript Tradition of Dudo of SaintQuentin's Gesta Normannorum», Anglo-Norman Studies, 6, 1983, 1984, p. 122-135.

KUPPER, Jean-Louis, Liège et l'Église Impériale aux XI ${ }^{e}$-XII ${ }^{e}$ siècles, Paris, Les BellesLettres, 1981, $568 \mathrm{p}$.

L'abbaye de Gorze au Xe siècle, Michel PARISSE et Otto Gerhard OEXLE (dir.), Nancy, Presses universitaires de Nancy (Lorraine), 1993, 248 p.

LAUER, Philippe, «Le manuscrit des Annales de Flodoard, reg. lat. 633 du Vatican », Mélanges d'Archéologie et d'Histoire, 18, Rome, École Française de Rome, 1898, p. 491-523 et planche XIII.

LAUER, Philippe, Le règne de Louis IV d'Outremer, Paris, Bouillon (Bibliothèque de l'École des Hautes Études, 127), 1900, XXXVIII-375 p.

LE JAN, Régine, Famille et pouvoir dans le monde Franc (VII ${ }^{e} X^{e}$ siècle), Paris, Publications de la Sorbonne, 1995, $571 \mathrm{p}$.

LE JAN, Régine, Histoire de la France: origines et premier essor 480-1180, Paris, Hachette (Carré Histoire), 1996, 254 p.

LE MAHO, Jacques, «La production éditoriale à Jumièges vers le milieu du X $\mathrm{X}^{\mathrm{e}}$ siècle», Tabularia «Études», n 1, 2001, p. 11-32.

LIFSHITZ, Felice, version électronique de Dudon: http://www.the-orb.net/ orb_done/dudo/dudintro.html

LOT, Ferdinand, Les derniers carolingiens: Lothaire - Louis V-Charles de Lorraine (954-991), Paris, Bouillon (Bibliothèque de l'École des Hautes Études, 87), 1891, XLVIII-479 p.

LOT, Ferdinand, Étude sur le règne d'Hugues Capet et la fin du Xe siècle, Paris, Bouillon (Bibliothèque de l'École des Hautes Études, 147), 1903, XL-525 p.

PARISOT, Robert, Les origines de la Haute-Lorraine et sa première Maison ducale (959-1033), Paris, Picard, 1909, 614 p.

PARISOT, Robert, Histoire de Lorraine, Paris, Picard, 1919, 520 p. 
PARISSE, Michel, La Noblesse lorraine, XI ${ }^{e}$-XIII ${ }^{e}$ siècle, Paris, Champion, 1976, 2 vol., LXVIII-1084 p.

PARISSE, Michel, L'abbaye de Gorze au Xe siècle, Nancy, Presses universitaires de Nancy, 1993, 247 p.

Poull, Georges, La Maison ducale de Lorraine, devenue la Maison impériale et royale d'Autriche, de Hongrie et de Bohème, Nancy, Presses universitaires de Nancy, 1991, 591-XXVIII p.

Poull, Georges, La Maison souveraine et ducale de Bar, Paris, Presses universitaires de Nancy, 1994, 455 p.

PRENTOUT, Henri, Étude critique sur Dudon de Saint-Quentin et son histoire des premiers ducs normands, Paris, Picard, 1916, XXXII-490 p.

RaOul Glaber, Histoires, prés. et trad. Mathieu ArnouX, Turnhout, Brepols (Miroir du Moyen-Âge), 1997, 323 p.

RAOUl GLABER, Les cinq livres de ses histoires (900-1044), éd. Maurice PROU, Paris, Picard (Collection de textes pour servir à l'étude et à l'enseignement de l'histoire), 1886, XV-143 p.

Recueil des Actes de Lothaire et Louis V, rois de France (954 - 987), éd. Louis HALPHEN, Paris, Académie des inscriptions et des belles-lettres (Chartes et diplômes relatifs à l'histoire de France), 1908, LVI + 231 p.

Recueil des Actes de Louis IV, roi de France (936-954), éd. Maurice Prou et Philippe LAUER, Paris, Académie des inscriptions et belles-lettres (Chartes et diplômes relatifs à l'histoire de France), 1914, LXXV-151 p.

RICHER, Histoire de France, éd. et trad. Robert Latouche, Paris, Champion (Les classiques de l'histoire de France au Moyen-Âge, 12), 1930-1937, 2 vol., XVII-692 p.

SAINT-DENIS, Alain, «Laon du XI ${ }^{\mathrm{e}}$ au XVe siècle», in Histoire de Laon et du Laonnois, Michel Bur (dir.), Toulouse, Privat, 1987, p. 63-135.

SASSIER, Yves, Hugues Capet : naissance d'une dynastie, Paris, Fayard, 1987, 357 p.

SHOpкOw, Leah, «The Carolinigian world of Dudo of Saint-Quentin », Journal of Medieval History, 15, 1989, p. 19-37.

SOT, Michel, Un historien et son Eglise au Xe siècle: Flodoard de Reims, Paris, Fayard, 1993, 832 p. 